\title{
Dual-Tree Complex Wavelet Transform in the Frequency Domain and an Application to Signal Classification
}

\author{
Julia Neumann Gabriele Steidl \\ Dept. of Mathematics and Computer Science \\ University of Mannheim \\ D-68131 Mannheim, Germany \\ $\{$ jneumann, steidl\}@uni-mannheim.de
}

September 17, 2003

\begin{abstract}
We examine Kingsbury's dual-tree complex wavelet transform in the frequency domain, where it can be formulated for standard wavelet filters without special filter design and apply the method to the classification of signals.

The obtained transforms achieve low shift sensitivity and better directionality compared to the real discrete wavelet transform while retaining the perfect reconstruction property.
\end{abstract}

\section{Introduction}

Wavelet techniques are successfully applied to various problems in signal and image processing. Data compression [2], motion estimation [1], texture synthesis [21], segmentation, classification [27] and denoising [9] are only some examples. In most applications special properties of the wavelets like symmetry, smoothness, shape or the number of vanishing moments are useful or even necessary. Best basis approaches [5, 22], [29, Chap. 8] or shape adaptation procedures [26] were designed to find optimal wavelet bases or frames for the application at hand.

However, a major problem of the common decimated discrete wavelet transform is its lack of shift invariance. This means that on shifts of the input signal, the wavelet coefficients vary substantially. The signal information may even not be stationary in the subbands so that the energy distribution across the subbands may change $[24,16]$.

The shift dependence is illustrated in Fig. 1 as also done in [24]. For presentation purposes, we chose a dilated Daubechies wavelet with three vanishing moments in Fig. 1 (a) as signal. Making a wavelet transform with itself, the result is clearly a single non-zero coefficient resulting in a single subband with positive energy in Fig. 1 (c). For later comparison purposes, we only plotted the coefficients' absolute value. Now on a signal shift of one sample to the right (Fig. 1 (e)), the other subbands in (f) and (h) also contain a significant portion of the signal energy. This shows that the orthogonal discrete wavelet transform is highly sensible to the signal alignment relative to the subsampling points.

To overcome the problem of shift dependence, one possible approach is to simply omit the subsampling that causes the shift dependence. In $m$ dimensions this introduces a redundancy of at least $1+d\left(2^{m}-1\right): 1$ for $d$ decomposition levels (in non-standard form). As a result, the coefficients are completely shift invariant in that they undergo the same shift as the input signal, 


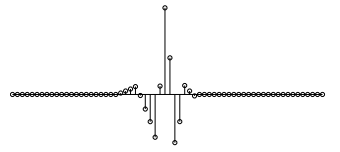

(a)

(b)

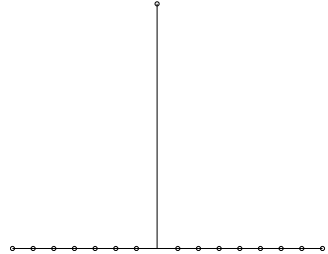

(c)

(d)

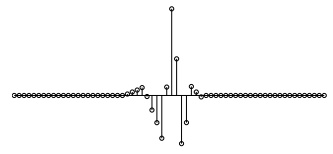

(e)

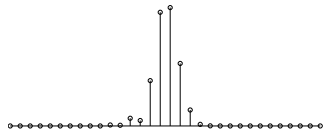

(f)

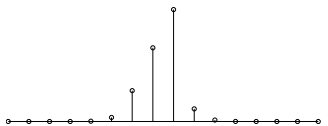

$(\mathrm{g})$

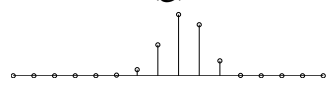

(h)

Figure 1: shift sensitivity of the discrete wavelet transform: (a) original signal, (b)-(d) magnitude of wavelet subband coefficients, (e) signal (a) shifted by one sample, (f)-(h) magnitude of new wavelet subband coefficients

but under a high cost that is often not desirable in signal processing. Techniques which omit or partially omit subsampling are also known as cycle spinning [4], oversampled filter banks [6] or undecimated wavelet transforms [18].

As an alternative, Kingsbury proposed a wavelet transform [16, 17, 14] that achieves approximate shift invariance with a redundancy of only $2^{m}: 1$ and showed some applications for motion estimation and denoising [17], texture synthesis [14] and retrieval [8]. The transform yields complex wavelet coefficients via a 'dual-tree' of parallel real filter banks. Another advantage of this complex wavelet transform is its directional selectivity in more dimensions that comes out without explicitly rotating a filter as in a Gabor filter bank, for example. However, if one wants to work with two real filter banks in the time domain, a special filter design is necessary which may not be the best with respect to the intended application.

In this paper we examine the dual-tree complex wavelet transform in the frequency domain where the known wavelet filters can be used and no special filter design is necessary. Of course this requires the application of fast Fourier transforms (FFTs) so that with respect to the arithmetic complexity this approach can only compete with real filter banks in the time domain having not too small filter lengths.

Organisation of the paper. In Sec. 2 we review Kingsbury's approach to achieve translation invariant combined filter banks in a sophisticated way. Sec. 3 proves that the construction proposed by Kingsbury indeed leads to wavelets with vanishing negative frequency parts. After explaining how the wavelet transform works in the frequency domain in Sec. 4, we point out the generalisation to higher dimensions in Sec. 5. Numerical examples illustrating the behaviour of the dual-tree complex wavelet transform for some standard wavelets with respect to shift invariance and rotation invariance are given in Sec. 6. Finally, Sec. 7 shows how the proposed transforms are suited to solve a signal classification problem. Sec. 8 summarises our findings. 


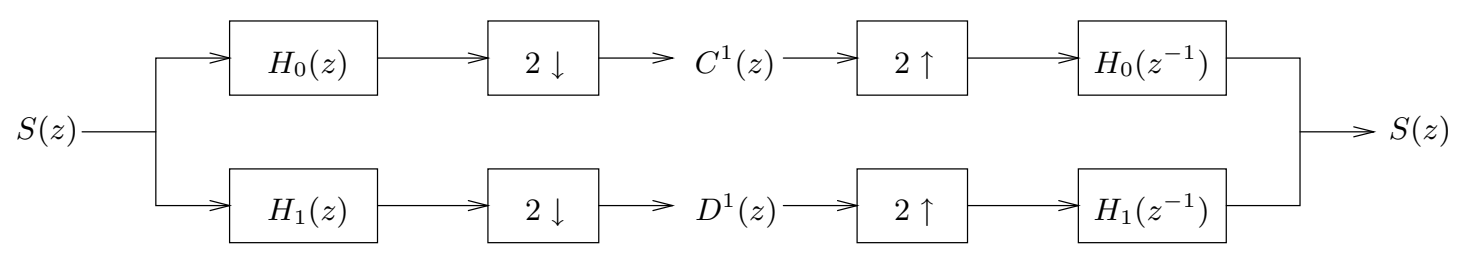

Figure 2: orthonormal filter bank

\section{Translation Invariance by Parallel Filter Banks}

We are interested in orthogonal two-channel filter banks with analysis low-pass filter given by the $z$-transform $H_{0}(z)=\sum_{k \in \mathbb{Z}} h_{0}[k] z^{-k}$, analysis high-pass filter $H_{1}(z)=\sum_{k \in \mathbb{Z}} h_{1}[k] z^{-k}$ and with synthesis filters $H_{0}\left(z^{-1}\right)$ and $H_{1}\left(z^{-1}\right)$. The corresponding filter bank is depicted in Fig. 2.

For an input signal $S(z)$, the analysis part of the filter bank inclusive subsequent upsampling produces the low-pass and the high-pass coefficients

$$
\begin{aligned}
C^{1}\left(z^{2}\right) & =\frac{1}{2}\left[S(z) H_{0}(z)+S(-z) H_{0}(-z)\right], \\
D^{1}\left(z^{2}\right) & =\frac{1}{2}\left[S(z) H_{1}(z)+S(-z) H_{1}(-z)\right],
\end{aligned}
$$

respectively, and decomposes the input signal into a low frequency part $S_{l}^{1}(z)$ and a high frequency part $S_{h}^{1}(z)$, more precisely

$$
S(z)=S_{l}^{1}(z)+S_{h}^{1}(z)
$$

where

$$
\begin{aligned}
S_{l}^{1}(z)=C^{1}\left(z^{2}\right) H_{0}\left(z^{-1}\right) & =\frac{1}{2}\left[S(z) H_{0}(z) H_{0}\left(z^{-1}\right)+S(-z) H_{0}(-z) H_{0}\left(z^{-1}\right)\right] \\
S_{h}^{1}(z)=D^{1}\left(z^{2}\right) H_{1}\left(z^{-1}\right) & =\frac{1}{2}\left[S(z) H_{1}(z) H_{1}\left(z^{-1}\right)+S(-z) H_{1}(-z) H_{1}\left(z^{-1}\right)\right] .
\end{aligned}
$$

Unfortunately, this decomposition is not shift invariant due to the second summands in (1) and (2), respectively, which were introduced by the down-/up-sampling operators. More precisely, if the input signal is shifted, say $z^{-1} S(z)$, the application of the filter bank results in the splitting

$$
z^{-1} S(z)=\tilde{S}_{l}^{1}(z)+\tilde{S}_{h}^{1}(z)
$$

where

$$
\tilde{S}_{l}^{1}(z)=\frac{1}{2} z^{-1}\left[S(z) H_{0}(z) H_{0}\left(z^{-1}\right)-S(-z) H_{0}(-z) H_{0}\left(z^{-1}\right)\right] \neq z^{-1} S_{l}^{1}(z)
$$

and similarly for the high-pass part. From this calculation one can see that the shift dependence is caused by the terms not containing $S(z)$, the so-called aliasing terms. Note that the filter bank is of course shift invariant with respect to a double shift since by $(-1)^{2}=1$ we have that $z^{-2} S(z)=z^{-2}\left(S_{l}^{1}(z)+S_{h}^{1}(z)\right)$.

One possibility to obtain a shift invariant decomposition consists in applying an additional filter bank with shifted analysis filters $z^{-1} H_{0}(z)$ and $z^{-1} H_{1}(z)$ and averaging the low- and the 


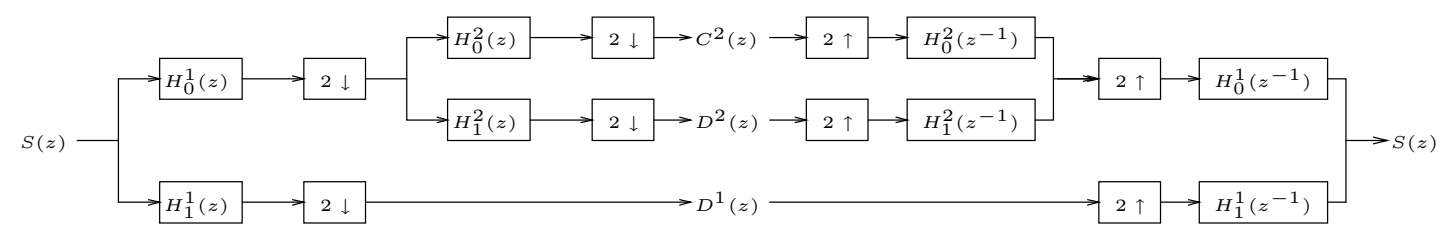

Figure 3: cascaded orthonormal filter bank

high-pass channels of both filter banks. If we signify the first filter bank by index $a$ and the second one by index $b$, then this procedure implies the decomposition

$$
S(z)=S_{l}^{1}(z)+S_{h}^{1}(z),
$$

where

$$
\begin{aligned}
S_{l}^{1}(z)= & \frac{1}{2}\left(C_{a}^{1}\left(z^{2}\right) H_{0 a}\left(z^{-1}\right)+C_{b}^{1}\left(z^{2}\right) H_{0 b}\left(z^{-1}\right)\right) \\
= & \frac{1}{4}\left[S(z)\left(H_{0}(z) H_{0}\left(z^{-1}\right)+H_{0}(z) H_{0}\left(z^{-1}\right)\right)\right. \\
& \left.\quad+S(-z)\left(H_{0}(-z) H_{0}\left(z^{-1}\right)-H_{0}(-z) H_{0}\left(z^{-1}\right)\right)\right] \\
= & \frac{1}{2} S(z) H_{0}(z) H_{0}\left(z^{-1}\right)
\end{aligned}
$$

and similarly for the high-pass part. The aliasing term containing $S(-z)$ in $S_{l}^{1}(z)$ has vanished and the decomposition becomes indeed shift invariant.

Iteration of the two-channel filter bank as shown in Fig. 3 for $J=2$ levels leads to a bandpass decomposition of the input signal. Note that the $j$ th filter bank may use its own filters $H^{j}$.

We are interested in cascaded filter banks with $J \geq 2$ levels. Let $A^{1}(z):=H_{0}^{1}(z), B^{1}(z):=$ $H_{1}^{1}(z)$ and

$$
\begin{aligned}
& A^{j}(z):=H_{0}^{1}(z) \cdots H_{0}^{j-1}\left(z^{2^{j-2}}\right) H_{0}^{j}\left(z^{2^{j-1}}\right) \\
& B^{j}(z):=H_{0}^{1}(z) \cdots H_{0}^{j-1}\left(z^{2^{j-2}}\right) H_{1}^{j}\left(z^{2^{j-1}}\right)
\end{aligned}
$$

for $j=2, \ldots, J$. Then the cascaded filter bank produces the low-pass coefficients

$$
C^{J}\left(z^{2^{J}}\right)=\frac{1}{2^{J}} \sum_{k=0}^{2^{J}-1} S\left(w_{2^{J}}^{k} z\right) A^{J}\left(w_{2^{J}}^{k} z\right)
$$

where $w_{m}:=e^{-2 \pi i / m}$ and for $j=1, \ldots, J$ the band-pass coefficients

$$
D^{j}\left(z^{2^{j}}\right)=\frac{1}{2^{j}} \sum_{k=0}^{2^{j}-1} S\left(w_{2^{j}}^{k} z\right) B^{j}\left(w_{2^{j}}^{k} z\right)=\frac{1}{2^{j}} \sum_{k=-2^{j-1}}^{2^{j-1}-1} S\left(w_{2^{j}}^{k} z\right) B^{j}\left(w_{2^{j}}^{k} z\right) .
$$


The input signal decomposes as

$$
\begin{aligned}
S(z) & =S_{l}^{J}(z)+\sum_{j=1}^{J} S_{h}^{j}(z) \\
& =C^{J}\left(z^{2^{J}}\right) A^{J}\left(z^{-1}\right)+\sum_{j=1}^{J} D^{j}\left(z^{2^{j}}\right) B^{j}\left(z^{-1}\right) \\
& =S_{l}^{J}(z)+\sum_{j=1}^{J} \frac{1}{2^{j}} \sum_{k=-2^{j-1}}^{2^{j-1}-1} S\left(w_{2^{j}}^{k} z\right) B^{j}\left(w_{2^{j}}^{k} z\right) B^{j}\left(z^{-1}\right) .
\end{aligned}
$$

Of course this decomposition is not shift invariant because the decomposition of $z^{-r} S(z)$ with $2^{j} \nmid r$ doesn't result in a $j$ th band-pass part $z^{-r} S_{h}^{j}(z)$ since $\left(w_{2^{j}}^{k}\right)^{-r} \neq 1$ for several $k$.

The ideal low-pass filter has the property supp $H_{0}\left(e^{2 \pi i \omega}\right)=\left[-\frac{1}{4}, \frac{1}{4}\right]$ for $\omega \in\left[-\frac{1}{2}, \frac{1}{2}\right]$. Here and in the following we write supp $f$ instead of supp $f \cap\left[-\frac{1}{2}, \frac{1}{2}\right]$ for a 1 -periodic function $f$. Let us assume that $H_{0}^{j}(z)$ fulfils

$$
\operatorname{supp} H_{0}^{j}\left(e^{2 \pi i \omega}\right) \subseteq\left[-\frac{1}{3}, \frac{1}{3}\right] \quad \forall j=1, \ldots, J
$$

for $\omega \in\left[-\frac{1}{2}, \frac{1}{2}\right]$. Then the corresponding orthogonal high-pass filters $H_{1}^{j}$ satisfy

$$
\operatorname{supp} H_{1}^{j}\left(e^{2 \pi i \omega}\right) \subseteq\left[-\frac{1}{2},-\frac{1}{6}\right] \cup\left[\frac{1}{6}, \frac{1}{2}\right] \quad \forall j=1, \ldots, J .
$$

In the following sections we will restrict ourselves to the QMF setting $H_{1}^{j}(z)=z^{-1} H_{0}^{j}\left(-z^{-1}\right)$ although the filter sign and translation do not affect our principal results. From (7) and (8) it follows for $j \geq 2$ that

$$
\begin{aligned}
& \operatorname{supp} A^{j}\left(e^{ \pm 2 \pi i \omega}\right) \subseteq\left[-\frac{1}{3 \cdot 2^{j-1}}, \frac{1}{3 \cdot 2^{j-1}}\right], \\
& \operatorname{supp} B^{j}\left(e^{ \pm 2 \pi i \omega}\right) \subseteq\left[-\frac{4}{3 \cdot 2^{j}},-\frac{1}{3 \cdot 2^{j}}\right] \cup\left[\frac{1}{3 \cdot 2^{j}}, \frac{4}{3 \cdot 2^{j}}\right] .
\end{aligned}
$$

Fig. 4 illustrates the support properties for $J=3$.

Let $x \bmod 1 \in\left[-\frac{1}{2}, \frac{1}{2}\right)$ denote the symmetric residue of $x \in \mathbb{R}$ modulo 1 and $[\cdot, \cdot] \bmod 1$ the 'interval' with elements taken modulo 1 . Now $B^{j}\left(w_{2^{j}}^{k} e^{2 \pi i \omega}\right)=B^{j}\left(e^{2 \pi i\left(\omega-\frac{k}{2^{j}}\right)}\right)$ is a $(1-$ periodic) shift of $B^{j}\left(e^{2 \pi i \omega}\right)$ by $\frac{k}{2^{j}}$. Thus,

$$
\operatorname{supp} B^{j}\left(w_{2^{j}}^{k} e^{2 \pi i \omega}\right) \subseteq\left(\left[\frac{-4+3 k}{3 \cdot 2^{j}}, \frac{-1+3 k}{3 \cdot 2^{j}}\right] \cup\left[\frac{1+3 k}{3 \cdot 2^{j}}, \frac{4+3 k}{3 \cdot 2^{j}}\right]\right) \bmod 1
$$

and consequently

$$
\operatorname{supp} B^{j}\left(w_{2^{j}}^{k} e^{2 \pi i \omega}\right) B^{j}\left(e^{-2 \pi i \omega}\right) \subseteq \begin{cases}{\left[-\frac{4}{3 \cdot 2^{j}},-\frac{1}{3 \cdot 2^{j}}\right] \cup\left[\frac{1}{3 \cdot 2^{j}}, \frac{4}{3 \cdot 2^{j}}\right]} & k=0, \\ \pm\left[\frac{1}{3 \cdot 2^{j}}, \frac{2}{3 \cdot 2^{3}}\right] & k= \pm 1 \\ \pm\left[\frac{2}{3 \cdot 2^{j}}, \frac{4}{3 \cdot 2^{j}}\right] & k= \pm 2 \\ \emptyset & 3 \leq|k| \leq 2^{j-1}\end{cases}
$$




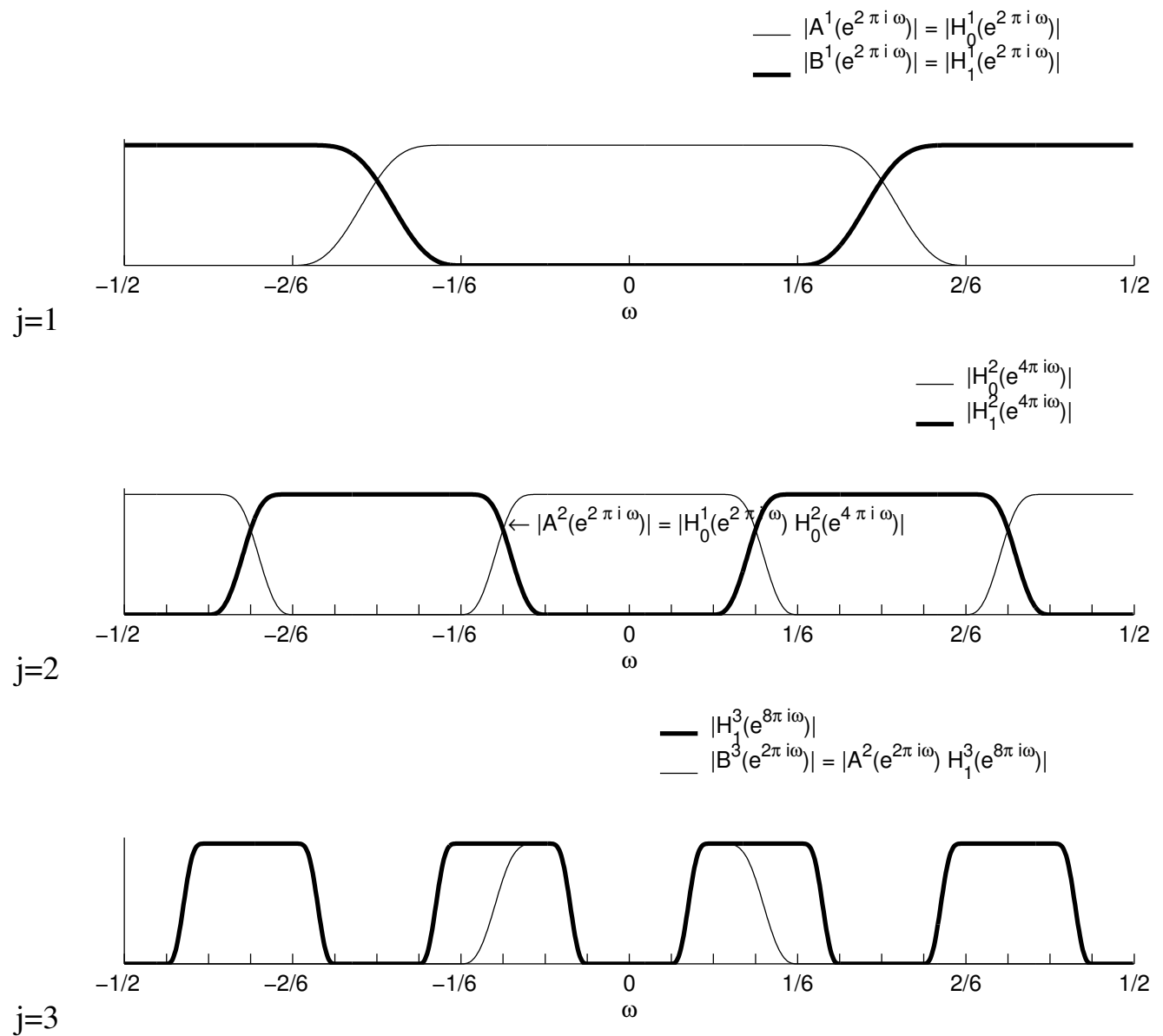

Figure 4: desired filter support properties at different levels $j$ 


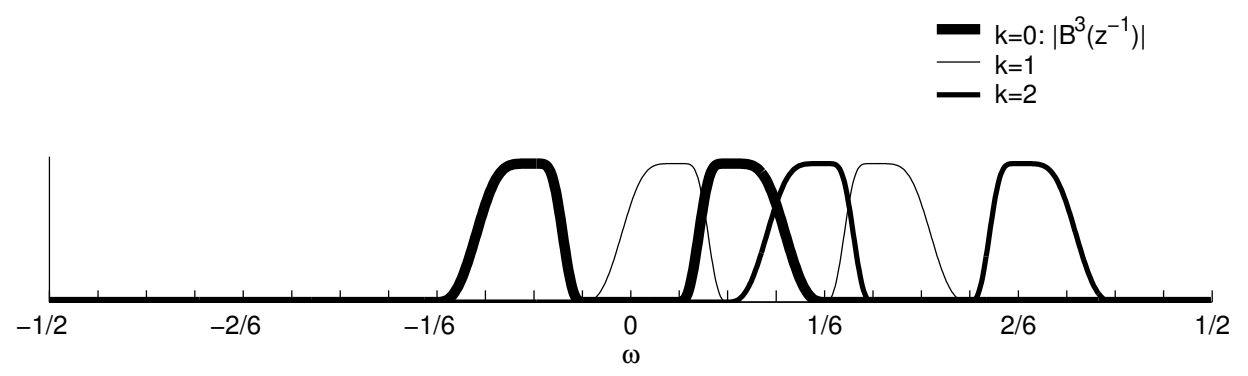

Figure 5: desired support of shifted high-pass filter $B^{j}\left(w_{2^{j}}^{k} e^{2 \pi i \omega}\right)$ at level $j=3$

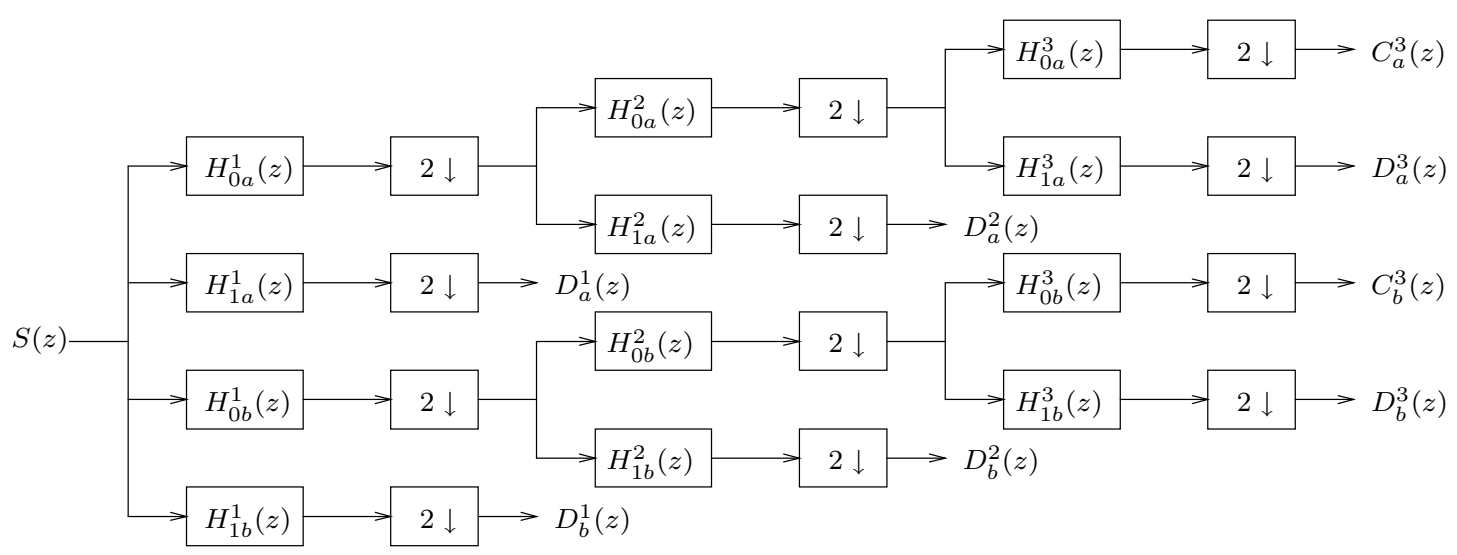

Figure 6: dual-tree filter bank

for $j \geq 2$. See Fig. 5 for an illustration. Hence the decomposition (6) can be rewritten as

$$
S(z)=S_{l}^{J}(z)+S_{h}^{1}(z)+\sum_{j=2}^{J} \frac{1}{2^{j}} \sum_{k=-2}^{2-\delta_{2 j}} S\left(w_{2^{j}}^{k} z\right) B^{j}\left(w_{2^{j}}^{k} z\right) B^{j}\left(z^{-1}\right) .
$$

To remedy the drawback of the decomposition being not shift invariant, Kingsbury suggested in $[14,16]$ to apply a 'dual-tree' of two parallel filter banks and combine their band-pass outputs as in the non-cascaded case. The structure of a resulting analysis filter bank is sketched in Fig.6, where we use again the index $a$ for the original cascaded filter bank and the index $b$ for the additional one. Then the input signal is split as

$$
\begin{aligned}
S(z)=\frac{1}{2}( & S_{l a}^{J}(z)+S_{l b}^{J}(z) \\
& \left.+\sum_{j=1}^{J} \frac{1}{2^{j}} \sum_{k=0}^{2^{j}-1} S\left(w_{2^{j}}^{k} z\right)\left(B_{a}^{j}\left(w_{2^{j}}^{k} z\right) B_{a}^{j}\left(z^{-1}\right)+B_{b}^{j}\left(w_{2^{j}}^{k} z\right) B_{b}^{j}\left(z^{-1}\right)\right)\right),
\end{aligned}
$$

where the inner sum can be restricted as in (10) if both $H_{0 a}$ and $H_{0 b}$ satisfy property (7).

For $P^{j}(z)=\sum_{k \in \mathbb{Z}} p^{j}[k] z^{-k}\left(p^{j}[k] \in \mathbb{C}\right)$, let $P^{j}(z)^{*}=\sum_{k \in \mathbb{Z}} \overline{p^{j}[k]} z^{k}$. Note that then $\operatorname{supp} P^{j}\left(e^{2 \pi i \omega}\right)=I$ implies $\operatorname{supp} P^{j}\left(e^{2 \pi i \omega}\right)^{*}=-I$. Let us assume that $B_{a}^{j}(z)$ and $B_{b}^{j}(z)$ have further the property that

$$
\begin{aligned}
& B_{a}^{j}(z)+i B_{b}^{j}(z)=P^{j}(z), \\
& B_{a}^{j}(z)-i B_{b}^{j}(z)=P^{j}(z)^{*},
\end{aligned}
$$


where $P^{j}$ is only supported on the positive frequencies $\omega \in\left[0, \frac{1}{2}\right]$. More precisely with respect to $(8)$ and (9b) we have

$$
\operatorname{supp} P^{j}\left(e^{2 \pi i \omega}\right) \subseteq\left[\frac{1}{3 \cdot 2^{j}}, \frac{4}{3 \cdot 2^{j}}\right]
$$

Obviously, (12) implies

$$
\begin{aligned}
& B_{a}^{j}(z)=\frac{1}{2}\left(P^{j}(z)+P^{j}(z)^{*}\right) \\
& B_{b}^{j}(z)=-\frac{1}{2} i\left(P^{j}(z)-P^{j}(z)^{*}\right) .
\end{aligned}
$$

Using these relations, we obtain in (11) that

$$
B_{a}^{j}\left(w_{2^{j}}^{k} z\right) B_{a}^{j}\left(z^{-1}\right)+B_{b}^{j}\left(w_{2^{j}}^{k} z\right) B_{b}^{j}\left(z^{-1}\right)=\frac{1}{2}\left(P^{j}\left(w_{2^{j}}^{k} z\right) P^{j}\left(z^{-1}\right)^{*}+P^{j}\left(w_{2^{j}}^{k} z\right)^{*} P^{j}\left(z^{-1}\right)\right) .
$$

Since $\operatorname{supp} P^{j}\left(w_{2^{j}}^{k} e^{2 \pi i \omega}\right) \subseteq\left[\frac{1+3 k}{3 \cdot 2^{j}}, \frac{4+3 k}{3 \cdot 2^{j}}\right] \bmod 1$ and $\operatorname{supp} P^{j}\left(e^{-2 \pi i \omega}\right)^{*}=\operatorname{supp} P^{j}\left(e^{2 \pi i \omega}\right) \subseteq$ $\left[\frac{1}{3 \cdot 2^{j}}, \frac{4}{3 \cdot 2^{j}}\right]$, this expression vanishes for $1 \leq|k| \leq 2^{j}-1$. If we further choose the filters $H_{\cdot a}^{1}$ and $H_{\cdot b}^{1}$ as in the non-cascaded case to cancel the aliasing at the first level, (11) can be rewritten as

$$
S(z)=\frac{1}{2}\left(S_{l a}^{J}(z)+S_{l b}^{J}(z)+\sum_{j=1}^{J} \frac{1}{2^{j}} S(z)\left(B_{a}^{j}(z) B_{a}^{j}\left(z^{-1}\right)+B_{b}^{j}(z) B_{b}^{j}\left(z^{-1}\right)\right)\right) .
$$

Of course, this band-pass decomposition is translation invariant. The complex filter $P^{j}$ from (12a) implies that the wavelet coefficients are combined in the same manner $D^{j}(z)=D_{a}^{j}(z)+$ $i D_{b}^{j}(z)$.

Similar ideas with the additional filter bank can be used for the alias cancellation of the lowpass filter. In this case, because of property (7), only the translated product filters for $k= \pm 1$ may cause aliasing. But as it is not easily attainable that the low-pass product filters have a property similar to (12), one cancels only the odd translates by letting the $b$ product filters be the $a$ filters shifted by half a sample.

Unfortunately, there don't exist real orthogonal FIR (finite impulse response) filters $H_{\cdot a}^{j}$ and $H_{\cdot b}^{j}$ such that $B_{a}^{j}$ and $B_{b}^{j}$ fulfil property (12). Therefore one can only construct FIR filters satisfying (12) approximately. Some special biorthogonal and orthogonal filters of this kind were constructed by Kingsbury $[15,16]$ and Selesnick et al. [23, 10].

\section{Construction of Filter Pairs in the Frequency Domain}

In this paper we propose to apply the cascaded filter banks in the frequency domain. This has the advantage that it suffices to know $H$. $\left(e^{2 \pi i \omega}\right)$ for some discrete values of $\omega$, while the explicit knowledge of the filter coefficients $h .[k]$ is not necessary. Hence, we can start with known orthogonal, but not necessarily FIR filters which approximately fulfil (7) and add an appropriate second filter bank. In our numerical experiments we apply, e.g., Butterworth filters [20,13] and orthogonal $B$-spline filters (Battle-Lemarié filters [3, Sec. 6.4]) of different orders.

Concerning the filter design, Kingsbury $[14,16]$ claims that the filters $H_{0 a}^{j}$ and $H_{0 b}^{j}$ at levels $j=2, \ldots, J$ should have a delay difference of half a sample: Suppose that we are given an orthogonal filter pair $H_{0}(z)$ and $H_{1}(z)=z^{-1} H_{0}\left(-z^{-1}\right)\left(z:=e^{2 \pi i \omega}\right)$. Then we will see that 
cascaded filter banks $a$ and $b$ such that (12) is fulfilled can be constructed in the following way: At level $j=1$, as proposed in the beginning of the previous section, we use the shifted filters

$$
\begin{aligned}
H_{0 a}^{1}(z) & :=H_{0}(z), & H_{1 a}^{1}(z) & :=H_{1}(z)=z^{-1} H_{0}\left(-z^{-1}\right), \\
H_{0 b}^{1}(z) & :=z^{-1} H_{0}(z), & H_{1 b}^{1}(z) & :=z^{-1} H_{1}(z)=z^{-2} H_{0}\left(-z^{-1}\right) .
\end{aligned}
$$

By the previous section, this guarantees that the combined band-pass component $S_{h}^{1}(z)$ is completely shift invariant.

At all higher levels $j=2, \ldots, J$ we use the filters

$$
H_{0 a}^{j}(z):=H_{0}(z), \quad H_{1 a}^{j}(z):=H_{1}(z)=z^{-1} H_{0}\left(-z^{-1}\right) .
$$

The filters in bank $b$ should differ from these filters by a shift of half a sample, i.e.

$$
H_{0 b}^{j}\left(e^{2 \pi i \omega}\right)=e^{-\pi i \omega} H_{0}\left(e^{2 \pi i \omega}\right) \quad \omega \in\left[-\frac{1}{2}, \frac{1}{2}\right) .
$$

This equals $z^{-\frac{1}{2}} H_{0}(z)$, but only for $z=e^{2 \pi i \omega}$ and $\omega \in\left[-\frac{1}{2}, \frac{1}{2}\right)$ since the right hand side is not 1 -periodic in $\omega$. Therefore we actually use its 1 -periodic extension

$$
H_{0 b}^{j}\left(e^{2 \pi i \omega}\right):=e^{-\pi i(\omega \bmod 1)} H_{0}\left(e^{2 \pi i \omega}\right) \quad \omega \in \mathbb{R} .
$$

In other words, the filter coefficients of $H_{0 b}^{j}$ are the Fourier coefficients of the 1-periodic function on the right hand side of (17). This function is not in $\mathcal{C}^{\infty}$, but in $\mathcal{C}^{m-1}$ if $H_{0}(z)=$ $(1+z)^{m} F(z)$.

As for $H_{0}(z)$ and $H_{1}(z)$, we define the high-pass filter by

$$
\begin{aligned}
H_{1 b}^{j}\left(e^{2 \pi i \omega}\right) & =e^{-2 \pi i \omega} H_{0 b}^{j}\left(e^{-2 \pi i\left(\omega+\frac{1}{2}\right)}\right) \\
& \stackrel{(17)}{=} e^{-2 \pi i \omega} e^{\pi i\left(\left(\omega+\frac{1}{2}\right) \bmod 1\right)} H_{0}\left(e^{-2 \pi i\left(\omega+\frac{1}{2}\right)}\right) \\
& =e^{\pi i\left(\left(\omega+\frac{1}{2}\right) \bmod 1\right)} H_{1}\left(e^{2 \pi i \omega}\right) \quad \omega \in \mathbb{R} .
\end{aligned}
$$

For $\omega \in\left[-\frac{1}{2}, \frac{1}{2}\right)$ this leads in particular to

$$
H_{1 b}^{j}\left(e^{2 \pi i \omega}\right)=\left\{\begin{aligned}
i e^{\pi i \omega} H_{1}\left(e^{2 \pi i \omega}\right) & \omega \in\left[-\frac{1}{2}, 0\right), \\
-i e^{\pi i \omega} H_{1}\left(e^{2 \pi i \omega}\right) & \omega \in\left[0, \frac{1}{2}\right) .
\end{aligned}\right.
$$

Note that $H_{0 b}$ and $H_{1 b}$ are supported as $H_{0}$ and $H_{1}$, respectively. In order to prove that our parallel filter banks satisfy (12), we need the following lemma:

Lemma 1. For $j \in \mathbb{N}$ and $\omega \in\left[-\frac{1}{2}, \frac{1}{2}\right)$, the function

$$
f_{j}(\omega):=\left(\left(2^{j} \omega+\frac{1}{2}\right) \bmod 1\right)-2 \omega-(2 \omega \bmod 1)-\cdots-\left(2^{j-1} \omega \bmod 1\right)
$$

fulfils

$$
f_{j}(\omega)=\left\{\begin{aligned}
\frac{1}{2} & \omega \in\left[-\frac{1}{2}, 0\right) \\
-\frac{1}{2} & \omega \in\left[0, \frac{1}{2}\right)
\end{aligned}\right.
$$


Proof. We will prove the relation by induction on $j$.

For $j=1$ we distinguish between two cases: For $\omega \in\left[0, \frac{1}{2}\right)$ we conclude that $2 \omega+\frac{1}{2} \in$ $\left[\frac{1}{2}, \frac{3}{2}\right)$ so that

$$
\left(2 \omega+\frac{1}{2}\right) \bmod 1-2 \omega=2 \omega-\frac{1}{2}-2 \omega=-\frac{1}{2} .
$$

For $\omega \in\left[-\frac{1}{2}, 0\right)$ we obtain $2 \omega+\frac{1}{2} \in\left[-\frac{1}{2}, \frac{1}{2}\right)$ so that

$$
\left(2 \omega+\frac{1}{2}\right) \bmod 1-2 \omega=2 \omega+\frac{1}{2}-2 \omega=\frac{1}{2} .
$$

If the assumption holds true for $k \leq j$, then we obtain

$$
\begin{aligned}
& f_{j+1}(\omega) \\
= & f_{j}(\omega)+\left(\left(2^{j+1} \omega+\frac{1}{2}\right) \bmod 1\right)-\left(\left(2^{j} \omega+\frac{1}{2}\right) \bmod 1\right)-\left(2^{j} \omega \bmod 1\right) \\
= & \left(\left(2^{j+1} \omega+\frac{1}{2}\right) \bmod 1\right)-\left(\left(2^{j} \omega+\frac{1}{2}\right) \bmod 1\right)-\left(2^{j} \omega \bmod 1\right)+\left\{\begin{aligned}
\frac{1}{2} & \omega \in\left[-\frac{1}{2}, 0\right), \\
-\frac{1}{2} & \omega \in\left[0, \frac{1}{2}\right) .
\end{aligned}\right.
\end{aligned}
$$

It remains to show that $\left(\left(2^{j+1} \omega+\frac{1}{2}\right) \bmod 1\right)-\left(\left(2^{j} \omega+\frac{1}{2}\right) \bmod 1\right)-\left(2^{j} \omega \bmod 1\right)=0$.

Every $\omega \in \mathbb{R}$ can be written as $\omega=k \cdot 2^{-(j+1)}+\omega_{0}$ for $k \in \mathbb{Z}$ and $\omega_{0} \in\left[0,2^{-(j+1)}\right)$. Then it follows

$$
\left(2^{j+1} \omega+\frac{1}{2}\right) \bmod 1=\left(k+2^{j+1} \omega_{0}+\frac{1}{2}\right) \bmod 1=2^{j+1} \omega_{0}-\frac{1}{2} .
$$

Concerning the remaining expressions, we consider again two cases:

$$
\begin{aligned}
\left(2^{j} \omega+\frac{1}{2}\right) \bmod 1 & =\left(\frac{k}{2}+2^{j} \omega_{0}+\frac{1}{2}\right) \bmod 1=\left\{\begin{array}{ll}
2^{j} \omega_{0}-\frac{1}{2} & 2 \mid k, \\
2^{j} \omega_{0} & 2 \nmid k
\end{array},\right. \\
2^{j} \omega \bmod 1 & =\left(\frac{k}{2}+2^{j} \omega_{0}\right) \bmod 1= \begin{cases}2^{j} \omega_{0} & 2 \mid k, \\
2^{j} \omega_{0}-\frac{1}{2} & 2 \nmid k\end{cases}
\end{aligned}
$$

so that

$\left(\left(2^{j+1} \omega+\frac{1}{2}\right) \bmod 1\right)-\left(\left(2^{j} \omega+\frac{1}{2}\right) \bmod 1\right)-\left(2^{j} \omega \bmod 1\right)=2^{j+1} \omega_{0}-\frac{1}{2}-2^{j} \omega_{0}+\frac{1}{2}-2^{j} \omega_{0}=0$.

By the following theorem, we will see that our filter banks fulfil indeed (12):

Theorem 2. Let the filters for two cascaded filter banks $a$ and $b$ be given by (15), (16), (17) and (18). For $j=2, \ldots, J$, let the corresponding product filters $B^{j}$. be defined by (3b). Then it holds

$$
B_{b}^{j}\left(e^{2 \pi i \omega}\right)=\left\{\begin{aligned}
i B_{a}^{j}\left(e^{2 \pi i \omega}\right) & \omega \in\left[-\frac{1}{2}, 0\right) \\
-i B_{a}^{j}\left(e^{2 \pi i \omega}\right) & \omega \in\left[0, \frac{1}{2}\right) .
\end{aligned}\right.
$$

Thus, if $B_{a}^{j}\left(e^{2 \pi i \omega}\right)$ and $B_{b}^{j}\left(e^{2 \pi i \omega}\right)$ are nearly supported as in (9b), $B_{a}^{j}\left(e^{2 \pi i \omega}\right) \pm i B_{b}^{j}\left(e^{2 \pi i \omega}\right)$ have the same support but only on the right or left hand side of the real axis, respectively. Together with the results of the previous section for the first level $j=1$, this means that if the filters are well localised, then the transform is approximately free of aliasing. 
Proof. First we obtain by (3b), (15a) and (16) that

$$
\begin{aligned}
B_{a}^{j}\left(e^{2 \pi i \omega}\right) & =H_{0}\left(e^{2 \pi i \omega}\right) H_{0}\left(e^{2 \cdot 2 \pi i \omega}\right) \cdots H_{0}\left(e^{2^{j-2} \cdot 2 \pi i \omega}\right) H_{1}\left(e^{2^{j-1} \cdot 2 \pi i \omega}\right) \\
& =U^{j}\left(e^{2 \pi i \omega}\right) H_{1}\left(e^{2^{j-1} \cdot 2 \pi i \omega}\right),
\end{aligned}
$$

where $U^{j}\left(e^{2 \pi i \omega}\right)=\prod_{k=0}^{j-2} H_{0}\left(e^{2^{k} \cdot 2 \pi i \omega}\right)$. On the other hand, we get by (3b), (15b), (17) and (18) that

$$
\begin{aligned}
B_{b}^{j}\left(e^{2 \pi i \omega}\right)= & e^{-2 \pi i \omega} H_{0}\left(e^{2 \pi i \omega}\right) \\
& e^{-\pi i(2 \omega \bmod 1)} H_{0}\left(e^{2 \cdot 2 \pi i \omega}\right) \cdots e^{-\pi i\left(2^{j-2} \omega \bmod 1\right)} H_{0}\left(e^{2^{j-2} \cdot 2 \pi i \omega}\right) H_{1 b}^{j}\left(e^{2^{j-1} \cdot 2 \pi i \omega}\right) \\
= & e^{-\pi i\left(2 \omega+(2 \omega \bmod 1)+\cdots+\left(2^{j-2} \omega \bmod 1\right)\right)} U^{j}\left(e^{2 \pi i \omega}\right) \\
& \cdot e^{\pi i\left(\left(2^{j-1} \omega+\frac{1}{2}\right) \bmod 1\right)} H_{1}\left(e^{2^{j-1} \cdot 2 \pi i \omega}\right) \\
= & e^{\pi i\left(\left(\left(2^{j-1} \omega+\frac{1}{2}\right) \bmod 1\right)-2 \omega-(2 \omega \bmod 1)-\cdots-\left(2^{j-2} \omega \bmod 1\right)\right)} B_{a}^{j}\left(e^{2 \pi i \omega}\right)
\end{aligned}
$$

and further by Lemma 1 that

$$
\begin{aligned}
B_{b}^{j}\left(e^{2 \pi i \omega}\right) & =B_{a}^{j}\left(e^{2 \pi i \omega}\right) \begin{cases}e^{\pi i / 2} & \omega \in\left[-\frac{1}{2}, 0\right), \\
e^{-\pi i / 2} & \omega \in\left[0, \frac{1}{2}\right),\end{cases} \\
& =B_{a}^{j}\left(e^{2 \pi i \omega}\right)\left\{\begin{aligned}
i & \omega \in\left[-\frac{1}{2}, 0\right) \\
-i & \omega \in\left[0, \frac{1}{2}\right)
\end{aligned}\right.
\end{aligned}
$$

This completes the proof.

One of the main ideas of our proof, namely the careful handling of the 1-periodicity of the filters we have later also found in Selesnick's paper [23]. However, Selesnick considered infinite filter iterations related to wavelets, i.e., in our notation $B^{j} .\left(z^{2^{1-j}}\right)$ for $j \rightarrow \infty$, where the $b$ filter bank including the first step has to be shifted by half a sample. In contrast, we address exactly the Kingsbury approach with a finite number of filter iterations and a special design of the first filter bank pair.

Besides the combined filter's strong orientation, another implication of the theorem is that the product filter $B_{b}^{j}$ is also real: If our basis filter $H_{0}$ is real, we obtain by (3b), (15a), (16) that the product filter $B_{a}^{j}$ is real. A filter is real if and only if its negative frequency response is the complex conjugate of its positive frequency response. Hence, Theorem 2 implies that $B_{b}^{j}$ is real if $B_{a}^{j}$ is real. Consequently, the wavelet coefficients of both trees $D_{a}^{j}$ and $D_{b}^{j}$ are real as well.

Finally, let us also have a look at the low-pass filters. If we also combine the low-pass filters as in (12a), the resulting filter still responds to negative frequencies. By (3a), (15), (16) and (17) we obtain that

$$
\begin{aligned}
& A_{a}^{j}\left(e^{2 \pi i \omega}\right)+i A_{b}^{j}\left(e^{2 \pi i \omega}\right) \\
= & H_{0}\left(e^{2 \pi i \omega}\right) \cdots H_{0}\left(e^{2^{j-1} \cdot 2 \pi i \omega}\right) \\
& +i e^{-2 \pi i \omega} e^{-\pi i(2 \omega \bmod 1)} \cdots e^{-\pi i\left(2^{j-1} \omega \bmod 1\right)} H_{0}\left(e^{2 \pi i \omega}\right) \cdots H_{0}\left(e^{2^{j-1} \cdot 2 \pi i \omega}\right) \\
= & A_{a}^{j}\left(e^{2 \pi i \omega}\right)\left(1+i e^{-\pi i\left(2 \omega+(2 \omega \bmod 1)+\cdots+\left(2^{j-1} \omega \bmod 1\right)\right)}\right) .
\end{aligned}
$$

For $\omega$ in the desired support $\left[-\frac{1}{3 \cdot 2^{j-1}}, \frac{1}{3 \cdot 2^{j-1}}\right]$ of the filters given in (9a), this simplifies to

$$
A_{a}^{j}\left(e^{2 \pi i \omega}\right)+i A_{b}^{j}\left(e^{2 \pi i \omega}\right)=A_{a}^{j}\left(e^{2 \pi i \omega}\right)\left(1+i e^{-2^{j} \pi i \omega}\right)
$$




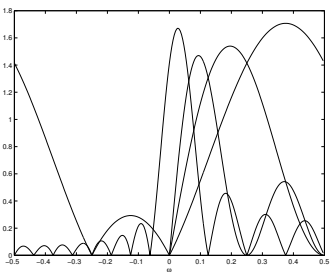

$\mathrm{H}$

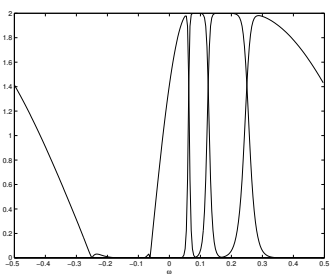

$\mathrm{BW}_{11}$

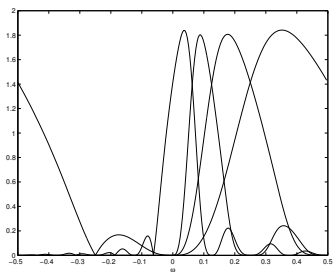

$\mathrm{D}_{3}$

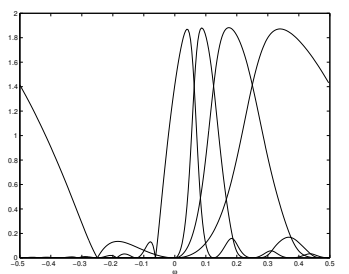

$\mathrm{BL}_{1}$

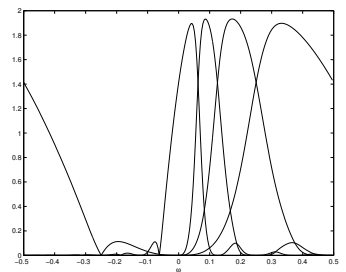

$\mathrm{BW}_{3}$

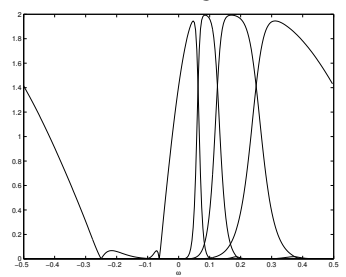

$\mathrm{BL}_{3}$

Figure 7: frequency response magnitude of complex filters $A_{a}^{3}(z)+i A_{b}^{3}(z)$ and $P^{j}(z)$ for $j=3,2,1$

so that

$$
\left|A_{a}^{j}\left(e^{2 \pi i \omega}\right)+i A_{b}^{j}\left(e^{2 \pi i \omega}\right)\right|=\left|A_{a}^{j}\left(e^{2 \pi i \omega}\right)\right|\left(2+2 \sin 2^{j} \pi \omega\right)^{\frac{1}{2}} .
$$

The second factor on the right hand side takes its minimum 0 at $\omega=-\frac{1}{2^{j+1}}$ and its maximum 2 at $\omega=\frac{1}{2^{j+1}}$. As a consequence, the frequency response of the combined low-pass filter also suppresses negative frequencies to some extent and leans to the right compared to $\left|A_{a}^{j}\left(e^{2 \pi i \omega}\right)\right|$. By a similar derivation, this second factor on the right hand side in (19) is also responsible for the negative frequency suppression of the first level high-pass filter $B_{a}^{1}\left(e^{2 \pi i \omega}\right)+i B_{b}^{1}\left(e^{2 \pi i \omega}\right)$.

By Theorem 2 we have an explicit construction method for cascaded filters with vanishing negative frequency parts. Their near shift invariance should improve the better condition (7) is fulfilled. In the next sections we will test filter banks based on the following standard filters $H_{0}$ : filter orthonormal basis filter $H_{0}$

$\begin{array}{cl}\mathrm{H} & \text { Haar filter } \\ \mathrm{D}_{3} & \text { Daubechies filter with } 3 \text { vanishing moments } \\ \mathrm{BW}_{m} & \text { Butterworth filter of order m [20, 13] } \\ \mathrm{BL}_{m} & \text { Battle-Lemarié filter of order m [3, Sec. 6.4] }\end{array}$

The Haar filter and the Daubechies filter are FIR filters of length two and six, respectively. The Butterworth and Battle-Lemarié orthogonal spline filters have infinite impulse response. Further, $\mathrm{BW}_{m}$ has $m$ vanishing moments and $\mathrm{BL}_{m}$ has $m+1$. For other properties see [20,3].

The frequency responses of the combined product filters for these basis filters are plotted in Fig. 7. As it was to expect, we observe that all complex filters $P^{j}(z)$ for $j \geq 2$ are only supported on the right half of the $\omega$-axis, whereas the low-pass and the first level filter $P^{1}(z)$ also respond to negative frequencies. Further, in agreement with (19), the low-pass filters' frequency responses all 'lean' to the right. The suppression of the sidelobes improves as the order $m$ of the filter increases and it fulfils property (7) more closely. But then, the filters become less concentrated in the time domain. 


\section{Fast Wavelet Transform in the Frequency Domain}

In the previous section we proposed a general construction method to obtain nearly shift invariant filter banks defined in the frequency domain and designed some exemplary filter banks. One possibility to apply these filter banks is to calculate the filter coefficients $h[k]$ from their Fourier coefficients $H\left(e^{2 \pi i \omega}\right)$ and then perform the usual filtering in the time domain. But as these filters will not have FIR due to their degraded regularity, the runtime of a time domain transform step becomes quadratic. Hence we have to make the decomposition in the frequency domain as also proposed in [11]. Note that this procedure imposes periodic boundary conditions which are very popular anyway. Moreover, we need FFTs for which there exist very efficient implementations, e.g., [12].

We will now explain the algorithm. Consider a given signal $s=\left(s_{0}, s_{1}, \ldots, s_{N-1}\right)$ of length $N$ in the time domain. In order to express or actually circumvent the down-sampling operation in the frequency domain, we rely on the polyphase representation [25, Sec. 4.2] of our quantities. After a $z$-transform, our signal reads

$$
S(z)=\sum_{j=0}^{N-1} s_{j} z^{-j}=S_{0}\left(z^{2}\right)+z^{-1} S_{1}\left(z^{2}\right),
$$

where $S_{0}$ comprises the even $z$-powers and $S_{1}$ the odd ones by

$$
\begin{aligned}
& S_{0}\left(z^{2}\right)=\frac{S(z)+S(-z)}{2}, \\
& S_{1}\left(z^{2}\right)=\frac{S(z)-S(-z)}{2} z .
\end{aligned}
$$

Similarly, we represent the filters for $i=0,1$ as

$$
H_{i}(z)=\sum_{j=0}^{N-1} h_{i}[j] z^{-j}=H_{i 0}\left(z^{2}\right)+z H_{i 1}\left(z^{2}\right)
$$

with

$$
\begin{aligned}
H_{i 0}\left(z^{2}\right) & =\frac{H_{i}(z)+H_{i}(-z)}{2}, \\
H_{i 1}\left(z^{2}\right) & =\frac{H_{i}(z)-H_{i}(-z)}{2} z^{-1} .
\end{aligned}
$$

Concerning the filter bank analysis depicted in the left part of Fig. 2, we obtain for $i=0,1$ that

$$
\begin{aligned}
S(z) H_{i}(z) & =\left[S_{0}\left(z^{2}\right)+z^{-1} S_{1}\left(z^{2}\right)\right]\left[H_{i 0}\left(z^{2}\right)+z H_{i 1}\left(z^{2}\right)\right] \\
S(-z) H_{i}(-z) & =\left[S_{0}\left(z^{2}\right)-z^{-1} S_{1}\left(z^{2}\right)\right]\left[H_{i 0}\left(z^{2}\right)-z H_{i 1}\left(z^{2}\right)\right]
\end{aligned}
$$

and further in (1)

$$
\begin{aligned}
& C^{1}\left(z^{2}\right)=S_{0}\left(z^{2}\right) H_{00}\left(z^{2}\right)+S_{1}\left(z^{2}\right) H_{01}\left(z^{2}\right) \\
& D^{1}\left(z^{2}\right)=S_{0}\left(z^{2}\right) H_{10}\left(z^{2}\right)+S_{1}\left(z^{2}\right) H_{11}\left(z^{2}\right)
\end{aligned}
$$

so that

$$
\left(\begin{array}{l}
C^{1}\left(z^{2}\right) \\
D^{1}\left(z^{2}\right)
\end{array}\right)=\left(\begin{array}{ll}
H_{00}\left(z^{2}\right) & H_{01}\left(z^{2}\right) \\
H_{10}\left(z^{2}\right) & H_{11}\left(z^{2}\right)
\end{array}\right)\left(\begin{array}{l}
S_{0}\left(z^{2}\right) \\
S_{1}\left(z^{2}\right)
\end{array}\right)
$$


where the matrix on the right hand side of the equation is called polyphase matrix of the analysis filter bank.

Now that we have an explicit decomposition formula in terms of the signals' polyphase components $S_{0}$ and $S_{1}$, we want to apply the decomposition on our discrete signal. We therefore calculate its discrete Fourier transform. Let $\hat{x}$ denote the Fourier transform of a signal $x$, then this reads with $z:=e^{2 \pi i k / N}=w_{N}^{-k}$

$$
\hat{s}_{k}:=S\left(e^{2 \pi i k / N}\right)=\sum_{j=0}^{N-1} s_{j} e^{-2 \pi i j k / N} \quad k=0, \ldots, N-1
$$

which requires a real FFT of length $N$. For the polyphase components, it holds

$$
S_{0}\left(z^{2}\right)=S_{0}\left(e^{2 \pi i k /(N / 2)}\right)=\hat{s}_{0 k} \stackrel{(20 \mathrm{a})}{=} \frac{1}{2}\left(S\left(e^{2 \pi i k / N}\right)+S\left(e^{2 \pi i\left(k+\frac{N}{2}\right) / N}\right),\right.
$$

analogously for $S_{1}$. Thus they may be calculated in the frequency domain by

$$
\hat{s}_{0 k}=\frac{1}{2}\left(\hat{s}_{k}+\hat{s}_{k+\frac{N}{2}}\right), \quad \hat{s}_{1 k}=\frac{1}{2} e^{2 \pi i k / N}\left(\hat{s}_{k}-\hat{s}_{k+\frac{N}{2}}\right) \quad k=0, \ldots, \frac{N}{2}-1
$$

which requires $N$ additions and $\frac{N}{2}$ multiplications of complex numbers. Finally, according to (22) the first decomposition step reads

$$
\begin{array}{r}
\left(\begin{array}{l}
C^{1}\left(e^{2 \pi i k /(N / 2)}\right) \\
D^{1}\left(e^{2 \pi i k /(N / 2)}\right)
\end{array}\right)=\left(\begin{array}{ll}
H_{00}\left(e^{2 \pi i k /(N / 2)}\right) & H_{01}\left(e^{2 \pi i k /(N / 2)}\right) \\
H_{10}\left(e^{2 \pi i k /(N / 2)}\right) & H_{11}\left(e^{2 \pi i k /(N / 2)}\right)
\end{array}\right)\left(\begin{array}{l}
S_{0}\left(e^{2 \pi i k /(N / 2)}\right) \\
S_{1}\left(e^{2 \pi i k /(N / 2)}\right)
\end{array}\right) \\
k=0, \ldots, \frac{N}{2}-1
\end{array}
$$

which requires $4 \frac{N}{2}$ multiplications and $2 \frac{N}{2}$ additions of complex numbers. For reconstructing the coefficients in the time domain, an inverse FFT of length $\frac{N}{2}$ is necessary for both the lowpass and the high-pass coefficients, respectively.

The polyphase components of the filters which appear in the polyphase matrix in (25) may be precomputed similar to $S_{0}$ and $S_{1}$ : From (21) it follows for $i=0$ for example

$$
\begin{array}{ll}
H_{00}\left(e^{2 \pi i k /(N / 2)}\right)=\frac{1}{2}\left[H_{0}\left(e^{2 \pi i k / N}\right)+H_{0}\left(e^{2 \pi i\left(k+\frac{N}{2}\right) / N}\right)\right] & k=0, \ldots, \frac{N}{2}-1, \\
H_{01}\left(e^{2 \pi i k /(N / 2)}\right)=\frac{1}{2} e^{-2 \pi i k / N}\left[H_{0}\left(e^{2 \pi i k / N}\right)-H_{0}\left(e^{2 \pi i\left(k+\frac{N}{2}\right) / N}\right)\right] & k=0, \ldots, \frac{N}{2}-1
\end{array}
$$

and further

$$
\begin{aligned}
\hat{h}_{00}[k] & =\frac{1}{2}\left(\hat{h}_{0}[k]+\hat{h}_{0}\left[k+\frac{N}{2}\right]\right) & k & =0, \ldots, \frac{N}{2}-1, \\
\hat{h}_{01}[k] & =\frac{1}{2} e^{-2 \pi i k / N}\left(\hat{h}_{0}[k]-\hat{h}_{0}\left[k+\frac{N}{2}\right]\right) & k & =0, \ldots, \frac{N}{2}-1 .
\end{aligned}
$$

Thereby, one has to take into account the $N$-periodisation of the shifted filters (17), (18) originally defined in the frequency range $\left[-\frac{1}{2}, \frac{1}{2}\right)$ which for $j=2, \ldots, J$ read

$$
\begin{aligned}
& H_{0 b}^{j}\left(e^{2 \pi i k / N}\right)= \begin{cases}e^{-\pi i k / N} H_{0}\left(e^{2 \pi i k / N}\right) & k=0, \ldots, \frac{N}{2}-1, \\
e^{-\pi i(k-N) / N} H_{0}\left(e^{2 \pi i k / N}\right) & k=\frac{N}{2}, \ldots, N-1,\end{cases} \\
& H_{1 b}^{j}\left(e^{2 \pi i k / N}\right)= \begin{cases}-i e^{\pi i k / N} H_{1}\left(e^{2 \pi i k / N}\right) & k=0, \ldots, \frac{N}{2}-1, \\
i e^{\pi i(k-N) / N} H_{1}\left(e^{2 \pi i k / N}\right) & k=\frac{N}{2}, \ldots, N-1\end{cases} \\
& =-i e^{\pi i k / N} H_{1}\left(e^{2 \pi i k / N}\right) \quad k=0, \ldots, N-1 .
\end{aligned}
$$


The further steps $j=2, \ldots, J$ are applied on the low-pass components $C^{j-1}$ whereat the signal lengths halve at each step as

$$
\begin{array}{r}
\left(\begin{array}{l}
C^{j}\left(e^{2 \pi i k /\left(N / 2^{j}\right)}\right) \\
D^{j}\left(e^{2 \pi i k /\left(N / 2^{j}\right)}\right)
\end{array}\right)=\left(\begin{array}{ll}
H_{00}\left(e^{2 \pi i k /\left(N / 2^{j}\right)}\right) & H_{01}\left(e^{2 \pi i k /\left(N / 2^{j}\right)}\right) \\
H_{10}\left(e^{2 \pi i k /\left(N / 2^{j}\right)}\right) & H_{11}\left(e^{2 \pi i k /\left(N / 2^{j}\right)}\right)
\end{array}\right)\left(\begin{array}{l}
C_{0}^{j-1}\left(e^{2 \pi i k /\left(N / 2^{j}\right)}\right) \\
C_{1}^{j-1}\left(e^{2 \pi i k /\left(N / 2^{j}\right)}\right)
\end{array}\right) \\
k=0, \ldots, \frac{N}{2^{j}}-1 .
\end{array}
$$

Note that the polyphase components $H_{00}\left(e^{2 \pi i k /\left(N / 2^{j}\right)}\right)=\hat{h}_{00}\left[2^{j-1} k\right]$ are already known from the previous steps. One can precompute the polyphase components or polyphase matrices. Once such precomputation for length $N$ was done the matrix values can be utilised for all signals of length $\frac{N}{2^{k}}$ where $k \in \mathbb{N}_{0}$.

If $N=2^{J}$ and we make a full decomposition in the frequency domain, the cost of the algorithm to obtain Fourier transformed wavelet coefficients in terms of number of complex multiplications reads

$$
\begin{array}{cc}
s \stackrel{(23)}{\longrightarrow} S(z) & \stackrel{(24),(25)}{\longrightarrow} C^{1}(z), D^{1}(z) \stackrel{(24),(26)}{\longrightarrow} C^{2}(z), D^{2}(z) \ldots \stackrel{(24),(26)}{\longrightarrow} C^{J}(z), D^{J}(z) \\
\frac{N}{2} \log _{2} N & \underbrace{+\frac{N}{2}+4 \frac{N}{2}+\frac{N}{4}+4 \frac{N}{4}+\cdots+1+4}_{5\left(\frac{N}{2}+\frac{N}{4}+\cdots+1\right)=5 N-1},
\end{array}
$$

i.e. $\frac{N}{2} \log _{2} N+5 N-1$ total. As the signal's polyphase components $S_{0}, S_{1}$ are nothing else than the sequence of even and odd numbered coefficients, respectively, one can also perform two FFTs of length $\frac{N}{2}$ on these two sequences which reduces the complexity further.

If the wavelet coefficients in the time domain are wanted, one additionally needs real IFFTs of lengths $\frac{N}{2}, \frac{N}{4}, \ldots, 2$ requiring altogether $2^{J-2} \log _{2} 2^{J-1}+2^{J-3} \log _{2} 2^{J-2}+\cdots+\log _{2} 2=$ $\sum_{j=1}^{J-1} 2^{j-1} j=2^{J-1}(J-2)+1=\frac{N}{2}\left(\log _{2} N-2\right)+1$ multiplications. Note that in particular in our application in Sec. 7 this back transform is not necessary.

Concerning the filter bank reconstruction depicted in the right part of Fig. 2, we obtain by (20) and (21) that

$$
\begin{aligned}
S_{0}\left(z^{2}\right) & =\frac{S(z)+S(-z)}{2} \\
& =\frac{1}{2}\left[C^{1}\left(z^{2}\right) H_{0}\left(z^{-1}\right)+D^{1}\left(z^{2}\right) H_{1}\left(z^{-1}\right)+C^{1}\left(z^{2}\right) H_{0}\left(-z^{-1}\right)+D^{1}\left(z^{2}\right) H_{1}\left(-z^{-1}\right)\right] \\
& =\frac{1}{2} C^{1}\left(z^{2}\right)\left[H_{0}\left(z^{-1}\right)+H_{0}\left(-z^{-1}\right)\right]+\frac{1}{2} D^{1}\left(z^{2}\right)\left[H_{1}\left(z^{-1}\right)+H_{1}\left(-z^{-1}\right)\right] \\
& =C^{1}\left(z^{2}\right) H_{00}\left(z^{-2}\right)+D^{1}\left(z^{2}\right) H_{10}\left(z^{-2}\right) \\
S_{1}\left(z^{2}\right) & =\frac{S(z)-S(-z)}{2} z \\
& =\frac{1}{2} z C^{1}\left(z^{2}\right)\left[H_{0}\left(z^{-1}\right)-H_{0}\left(-z^{-1}\right)\right]+\frac{1}{2} z D^{1}\left(z^{2}\right)\left[H_{1}\left(z^{-1}\right)-H_{1}\left(-z^{-1}\right)\right] \\
& =C^{1}\left(z^{2}\right) H_{01}\left(z^{-2}\right)+D^{1}\left(z^{2}\right) H_{11}\left(z^{-2}\right) .
\end{aligned}
$$

Consequently, the final reconstruction is simply

$$
\left(\begin{array}{l}
S_{0}\left(z^{2}\right) \\
S_{1}\left(z^{2}\right)
\end{array}\right)=\left(\begin{array}{ll}
H_{00}\left(z^{-2}\right) & H_{10}\left(z^{-2}\right) \\
H_{01}\left(z^{-2}\right) & H_{11}\left(z^{-2}\right)
\end{array}\right)\left(\begin{array}{l}
C^{1}\left(z^{2}\right) \\
D^{1}\left(z^{2}\right)
\end{array}\right)
$$

where further steps follow the same rule. 


\section{Complex Wavelet Transform in Higher Dimensions}

In order to extend the transform to higher-dimensional signals, a filter bank is usually applied separably in all dimensions. But for the complex filters, a further extension is necessary as also indicated in [17]:

If we apply the Fourier transform to a real signal, we obtain that the negative frequency part is just the complex conjugate of the positive frequency part, so that the signal may be recovered from just one half of its spectrum. Hence the signal is recoverable from the filter output of our complex filter banks with just positive frequency response filters. But in higher dimensions, only the opposite quadrant Fourier coefficients are redundant being complex conjugates. With the separably applied complex product filter having only frequency response in the positive quadrant, the signal hence cannot be recovered. In $m$ dimensions, the frequency response of $2^{m-1}$ non-opposite quadrants is necessary to recover the signal. All necessary quadrants are, e.g., covered by constructing all positive/negative frequency tensor products of the filters in $m-1$ dimensions and leaving the filter in the remaining dimension fixed. At level $j$, the filter bank for $m=2$ should then produce the outputs

$$
\begin{aligned}
C_{a / b}^{j}\left(z_{1}, z_{2}\right) & =\left(2^{j} \downarrow\right)\left(\left(A_{a}^{j}\left(z_{1}\right) \pm i A_{b}^{j}\left(z_{1}\right)\right)\left(A_{a}^{j}\left(z_{2}\right)+i A_{b}^{j}\left(z_{2}\right)\right) S\left(z_{1}, z_{2}\right)\right), \\
D_{1 a / b}^{j}\left(z_{1}, z_{2}\right) & =\left(2^{j} \downarrow\right)\left(\left(A_{a}^{j}\left(z_{1}\right) \pm i A_{b}^{j}\left(z_{1}\right)\right)\left(B_{a}^{j}\left(z_{2}\right)+i B_{b}^{j}\left(z_{2}\right)\right) S\left(z_{1}, z_{2}\right)\right), \\
D_{2 a / b}^{j}\left(z_{1}, z_{2}\right) & =\left(2^{j} \downarrow\right)\left(\left(B_{a}^{j}\left(z_{1}\right) \pm i B_{b}^{j}\left(z_{1}\right)\right)\left(A_{a}^{j}\left(z_{2}\right)+i A_{b}^{j}\left(z_{2}\right)\right) S\left(z_{1}, z_{2}\right)\right), \\
D_{3 a / b}^{j}\left(z_{1}, z_{2}\right) & =\left(2^{j} \downarrow\right)\left(\left(B_{a}^{j}\left(z_{1}\right) \pm i B_{b}^{j}\left(z_{1}\right)\right)\left(B_{a}^{j}\left(z_{2}\right)+i B_{b}^{j}\left(z_{2}\right)\right) S\left(z_{1}, z_{2}\right)\right),
\end{aligned},
$$

where $\left(2^{j} \downarrow\right)$ denotes down-sampling by $2^{j}$ and the subscript $a / b$ is related to the \pm in the first factor, see also Fig. 8. Hence, the filter bank has six complex high-pass subbands at each level and two complex low-pass subbands in contrast to three real high-pass and one real low-pass subband for the real two-dimensional transform. This shows that the complex transform has a coefficient redundancy of $4: 1$ or $2^{m}: 1$ in $m$ dimensions.

Due to the special filter construction and the dual-tree implementation in our case, the required product filters may be constructed easily [15]. For our filter pair subsumed in Theorem 2, we obtain a filter supported on the other half of the $\omega$-axis by just toggling the sign in $B_{a}^{j}\left(e^{2 \pi i \omega}\right) \pm i B_{b}^{j}\left(e^{2 \pi i \omega}\right)$ or combining the filter outputs of the separate filters $B_{a}^{j}$ and $B_{b}^{j}$ with a different sign, respectively. Fig. 8 shows two levels of the resulting dual-tree filter bank for a two-dimensional input signal $\mathbf{S}$ without subsampling operations. The markers indicate real coefficient parts $r$ and row or column imaginary parts $i_{1}$ and $i_{2}$, respectively. The output of each subband in the filter bank is a 4-tuple $(r, t, s, u) \hat{=} r+s i_{1}+t i_{2}+u i_{1} i_{2}$. To obtain the usual filters, one sets $i_{1}=i_{2}=i$ and obtains $(r-u)+i(s+t)$ for the $a$ coefficients, for example $D_{1 a}^{1}$. For the conjugate row filter, one sets $-i_{1}=i_{2}=i$ and obtains $(r+u)+i(-s+t)$ for the $b$ coefficients. This operation is indicated by the $\Sigma, \Delta$ blocks at the end of each subband. For further decomposition steps, the row and column filtering blocks in the second level can be iterated for the low-pass channel, i.e. they replace the uppermost $\Sigma, \Delta$ block. One can see in Fig. 8 that the real coefficients marked by $r$ then are transformed step by step by themselves without interleaving with the other coefficient bands. The same occurs for the other three components. Hence, in two dimensions, from level two on we have four parallel trees that are only combined in the end to build the complex coefficients.

In the case of real two-dimensional filter banks, the three high-pass filters have orientations of $0^{\circ}, 45^{\circ}$ and $90^{\circ}$, respectively. For the complex filters, Kingsbury $[14,16]$ claims that his six 


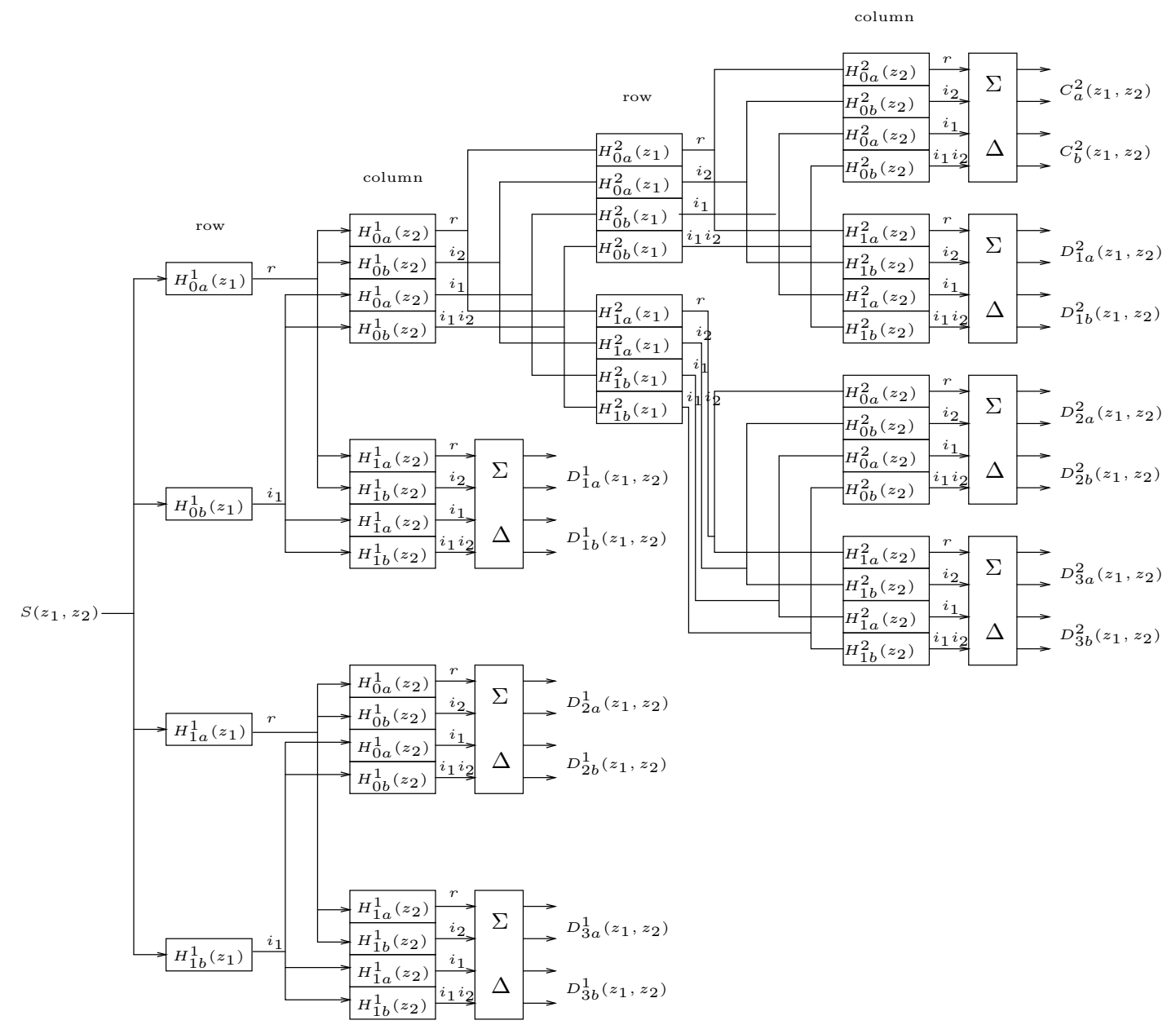

Figure 8: dual-tree filter bank for a 2D signal $\mathbf{S}$ without subsampling operations 


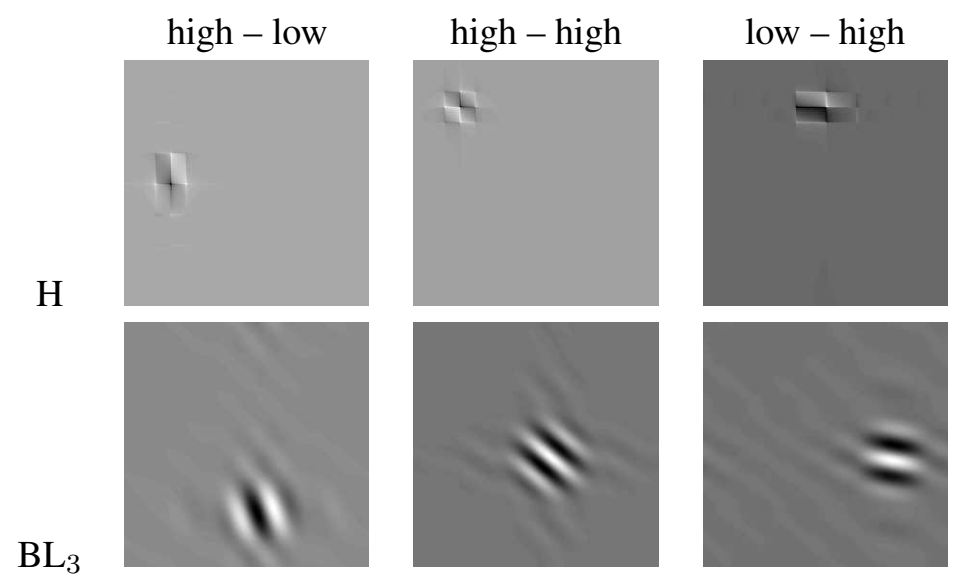

Figure 9: real 2D impulse response of complex (row - column) filters at level 5

subband filters are oriented at $\pm 15^{\circ}, \pm 45^{\circ}, \pm 75^{\circ}$. Indeed, if we approximate the filter orientation by the angle of the maximal frequency response magnitude, by $\max \left|F_{\text {row }}\left(z_{1}\right) F_{\text {col }}\left(z_{2}\right)\right|=$ $\left(\max \left|F_{\text {row }}\left(z_{1}\right)\right|\right)\left(\max \left|F_{\text {col }}\left(z_{2}\right)\right|\right)$ the orientation is roughly given by

$$
\arctan \frac{\arg \max _{\omega}\left|F_{\text {col }}\left(e^{2 \pi i \omega}\right)\right|}{\arg \max _{\omega}\left|F_{\text {row }}\left(e^{2 \pi i \omega}\right)\right|}
$$

so that we have to examine the maxima of $\left|A_{a}^{j}+i A_{b}^{j}\right|$ and $\left|B_{a}^{j}+i B_{b}^{j}\right|$. By Theorem 2 and (19), we see that they depend on the maxima of the single filters $B_{a}^{j}$ and $A_{a}^{j}$. As can already be guessed from Fig. 7, these depend on the basis filter and even on the level $j$. For example for the Haar filter $\mathrm{H}$ we have

$$
\begin{aligned}
A_{a}^{j}\left(e^{2 \pi i \omega}\right) & =\left(\frac{1+e^{-2 \pi i \omega}}{\sqrt{2}}\right) \cdots\left(\frac{1+e^{-2^{j-1} 2 \pi i \omega}}{\sqrt{2}}\right) \\
\Rightarrow\left|A_{a}^{j}\left(e^{2 \pi i \omega}\right)\right| & =2^{\frac{j}{2}}\left|\cos \pi \omega \cdots \cos 2^{j-1} \pi \omega\right| \stackrel{[7, p .211]}{=} 2^{-\frac{j}{2}}\left|\frac{\sin 2^{j} \pi \omega}{\sin \pi \omega}\right| .
\end{aligned}
$$

With (19), one can easily check that $\arg \max _{\omega}\left|A_{a}^{j}\left(e^{2 \pi i \omega}\right)+i A_{b}^{j}\left(e^{2 \pi i \omega}\right)\right| \neq 2^{-j} \cdot$ const, nor is the total orientation angle constant during the levels. The actual orientations for all our row high-pass and the column low-pass filters (corresponding to coefficients $D_{3 a}$ ) vary roughly from $15^{\circ}$ to $30^{\circ}$. Of course, not only the maximum frequency response is important for the filter's orientation, but the filter may be a superposition of differently oriented components as it can be seen in Fig. 9, where the real parts of some filter impulse responses are plotted. The corresponding complex parts look very similar and the other orientations are roughly conjugated mirrors.

Even if the complex transform does not have fixed filter orientations, we expect it to be more robust against two-dimensional disturbances because of the six differently oriented subbands.

\section{Invariance Performance}

In this section we examine the behaviour of the dual-tree wavelet transform based on the standard wavelets proposed at the end of Sec. 3 with respect to translation and rotation invariance. 


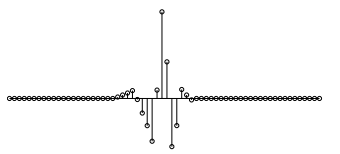

(a)

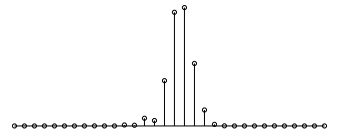

(b)

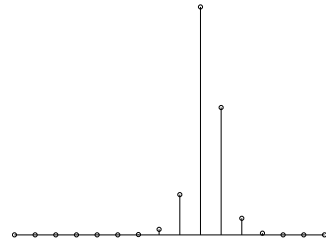

(c)

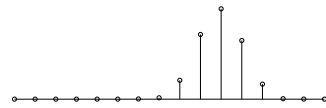

(d)

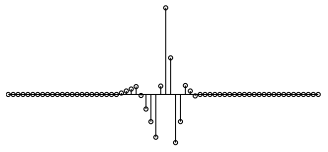

(e)

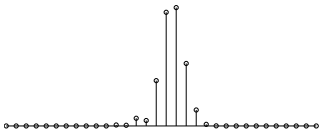

(f)

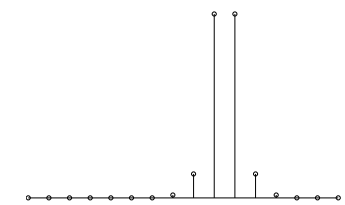

(g)

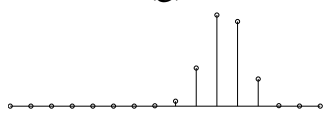

(h)

Figure 10: shift sensitivity of the complex transform in the frequency domain: (a) original signal, (b)-(d) magnitude of subband coefficients, (e) signal from (a) shifted by one sample, (f)-(h) magnitude of new subband coefficients

First we give an illustration of the shift invariance properties. Fig. 1 in Sec. 1 illustrates the shift dependence of the common discrete wavelet transform considering the $\mathrm{D}_{3}$ wavelet as an example. For the same basis filter $\mathrm{D}_{3}$, the dual-tree complex transform yields the results shown in Fig. 10. To reconstruct the coefficients in the time domain, we had to apply an inverse FFT as described in Sec. 4. Anyway, we are only able to plot the magnitude of the complex coefficients here. In contrast to the real transform, in this example the distribution of the coefficient energy across the subbands is almost equal for both signals. Even the shape of the coefficients mainly stays the same; they provide interpolability as required in [24]. As expected, the coefficient magnitudes at the first level come closest to shift invariance.

This illustration already indicates that the complex wavelet transform behaves more stable on signal shifts. To assess the influence of signal shifts more methodically, we compute the variance of the channel energies $\left\|D^{j}(z)\right\|_{2}$ with respect to all signal shifts $z^{k} S(z)$ $(k=1, \ldots, N)$ for a step signal s of length $N=256$. As the coefficients are real, the energy is $\left\|D_{a}^{j}(z)+i D_{b}^{j}(z)\right\|_{2}=\left(\left\|D_{a}^{j}(z)\right\|_{2}^{2}+\left\|D_{b}^{j}(z)\right\|_{2}^{2}\right)^{\frac{1}{2}}$. For this, no FFT back transform from the frequency domain is necessary since, by the Parseval identity, the 2-norms of the wavelet coefficients in the time and the frequency domain coincide. Table 1 shows the results for real (subsampled) transforms, our complex sample filters and Kingsbury's time domain dual-tree transform with 14-tap orthonormal filter and (9,7)-tap biorthogonal filters in the first step [16]. The coefficients for the complex and real transforms have the same magnitude as all filters $H_{0 a}(z), H_{1 a}(z)$ are normalised as well. Hence the variances are comparable. Evidently, the constructed complex filter banks are all less sensitive to signal shifts than the real transforms. The variance even gets relatively close to zero which is the energy variance for the non-subsampled transform. At level one of the complex transform, the variance always has to be zero because effectively, no subsampling is done due to the delayed filters from (15b). The shift invariance improves with the 


\begin{tabular}{c||c|c|c|c|c|c|c|c} 
level & 1 & 2 & 3 & 4 & 5 & 6 & 7 & 8 \\
filter & & & & & & & & \\
\hline H real & $2.5 \mathrm{e}-01$ & $2.5 \mathrm{e}-01$ & $3.8 \mathrm{e}-01$ & $6.9 \mathrm{e}-01$ & $1.3 \mathrm{e}+00$ & $2.7 \mathrm{e}+00$ & $5.3 \mathrm{e}+00$ & $5.3 \mathrm{e}+00$ \\
$\mathrm{D}_{3}$ real & $1.2 \mathrm{e}-02$ & $4.1 \mathrm{e}-02$ & $9.4 \mathrm{e}-02$ & $1.8 \mathrm{e}-01$ & $3.6 \mathrm{e}-01$ & $6.1 \mathrm{e}-01$ & $4.1 \mathrm{e}+00$ & $4.1 \mathrm{e}+00$ \\
\hline Kingsbury & & & & & & & & \\
$(9,7) / 14-$ tap & $1.6 \mathrm{e}-30$ & $6.3 \mathrm{e}-04$ & $5.3 \mathrm{e}-04$ & $4.2 \mathrm{e}-04$ & $1.0 \mathrm{e}-03$ & $9.2 \mathrm{e}-04$ & $4.0 \mathrm{e}-03$ & $4.0 \mathrm{e}-03$ \\
\hline $\mathrm{H}$ & $1.1 \mathrm{e}-31$ & $4.1 \mathrm{e}-02$ & $5.5 \mathrm{e}-02$ & $9.6 \mathrm{e}-02$ & $1.8 \mathrm{e}-01$ & $3.2 \mathrm{e}-01$ & $3.2 \mathrm{e}-01$ & $3.2 \mathrm{e}-01$ \\
$\mathrm{D}_{3}$ & $2.2 \mathrm{e}-29$ & $2.7 \mathrm{e}-03$ & $4.4 \mathrm{e}-03$ & $7.8 \mathrm{e}-03$ & $1.5 \mathrm{e}-02$ & $7.2 \mathrm{e}-02$ & $6.4 \mathrm{e}-02$ & $6.4 \mathrm{e}-02$ \\
$\mathrm{BW}_{3}$ & $1.6 \mathrm{e}-29$ & $4.3 \mathrm{e}-04$ & $7.5 \mathrm{e}-04$ & $1.4 \mathrm{e}-03$ & $2.7 \mathrm{e}-03$ & $8.4 \mathrm{e}-03$ & $1.4 \mathrm{e}-02$ & $1.4 \mathrm{e}-02$ \\
$\mathrm{BW}_{11}$ & $5.1 \mathrm{e}-30$ & $3.5 \mathrm{e}-11$ & $7.0 \mathrm{e}-11$ & $1.1 \mathrm{e}-10$ & $1.6 \mathrm{e}-10$ & $7.3 \mathrm{e}-11$ & $1.1 \mathrm{e}-08$ & $1.1 \mathrm{e}-08$ \\
$\mathrm{BL}_{1}$ & $1.0 \mathrm{e}-29$ & $1.7 \mathrm{e}-03$ & $2.9 \mathrm{e}-03$ & $5.3 \mathrm{e}-03$ & $1.1 \mathrm{e}-02$ & $3.1 \mathrm{e}-02$ & $3.7 \mathrm{e}-02$ & $3.7 \mathrm{e}-02$ \\
$\mathrm{BL}_{3}$ & $6.6 \mathrm{e}-30$ & $5.7 \mathrm{e}-06$ & $1.1 \mathrm{e}-05$ & $2.0 \mathrm{e}-05$ & $3.7 \mathrm{e}-05$ & $6.2 \mathrm{e}-05$ & $4.4 \mathrm{e}-04$ & $4.4 \mathrm{e}-04$
\end{tabular}

Table 1: energy variance on shifts of the step signal

\begin{tabular}{|c|c|c|c|c|c|c|c|c|c|c|}
\hline \multirow[b]{2}{*}{ filter } & \multicolumn{5}{|c|}{ low-pass } & \multicolumn{5}{|c|}{ high-pass } \\
\hline & 1 & 2 & 3 & 4 & 5 & 1 & 2 & 3 & 4 & 5 \\
\hline H real & -4.77 & -1.96 & -0.89 & -0.43 & -0.21 & -4.77 & 0.00 & 2.29 & 3.23 & 3.63 \\
\hline $\mathrm{D}_{3}$ real & -7.64 & -5.53 & -7.49 & -9.60 & -8.09 & -7.64 & -2.41 & 1.44 & -2.10 & -3.21 \\
\hline $\begin{array}{l}\text { Kingsbury } \\
(9,7) / 14 \text {-tap } \\
{[16, \text { Table 3] }}\end{array}$ & $-\infty$ & -23.19 & -29.33 & -28.56 & -28.57 & $-\infty$ & -21.81 & -18.96 & -24.85 & -24.15 \\
\hline $\mathrm{H}$ & $-\infty$ & -9.80 & -5.72 & -3.20 & -1.29 & $-\infty$ & -7.84 & -5.08 & -2.34 & -0.19 \\
\hline$D_{3}$ & $-\infty$ & -18.83 & -19.32 & -18.57 & -18.44 & $-\infty$ & -17.23 & -12.30 & -13.81 & -13.22 \\
\hline $\mathrm{BW}_{3}$ & $-\infty$ & -27.16 & -26.78 & -26.10 & -25.61 & $-\infty$ & -26.08 & -21.68 & -21.50 & -21.11 \\
\hline $\mathrm{BW}_{11}$ & $-\infty$ & -92.81 & -92.84 & -92.90 & -92.34 & $-\infty$ & -92.95 & -87.77 & -87.79 & -87.52 \\
\hline $\mathrm{BL}_{1}$ & $-\infty$ & -22.35 & -21.14 & -20.34 & -19.64 & $-\infty$ & -21.05 & -16.92 & -16.23 & -15.74 \\
\hline $\mathrm{BL}_{3}$ & $-\infty$ & -44.16 & -44.29 & -43.85 & -43.59 & $-\infty$ & -43.55 & -38.90 & -38.99 & -38.70 \\
\hline
\end{tabular}

Table 2: aliasing energy ratio $10 \log _{10} R_{\text {alias }}$ in $\mathrm{dB}$ at levels 1 to 5

order, and, therewith, the number of vanishing moments of the Butterworth and Battle-Lemarié wavelets because property (7) is better fulfilled. Both wavelet filters of order three are at least comparable to the large Kingsbury filter with respect to shift invariance. Note that the channel energies will be computed in our signal classification application so that it is important that they do not heavily depend on the signal alignment.

To quantify the effect of the aliasing causing the shift dependence more generally, we determine the aliasing energy ratio as done by Kingsbury in [16]. Considering equation (11), we already observed that the aliasing terms are the terms with $S\left(w_{2^{j}}^{k} z\right)$ for $k \neq 0$. Hence we determine the aliasing energy ratio for a subband at level $j$ as

$$
R_{\text {alias }}=\frac{\sum_{k=1}^{2^{j}-1}\left\|B_{a}^{j}\left(w_{2^{j}}^{k} z\right) B_{a}^{j}\left(z^{-1}\right)+B_{b}^{j}\left(w_{2^{j}}^{k} z\right) B_{b}^{j}\left(z^{-1}\right)\right\|_{2}}{\left\|B_{a}^{j}(z) B_{a}^{j}\left(z^{-1}\right)+B_{b}^{j}(z) B_{b}^{j}\left(z^{-1}\right)\right\|_{2}},
$$

where a filter $H(z)$ is regarded as a function $\omega \mapsto H\left(e^{2 \pi i \omega}\right) \in L_{2}\left(\left[-\frac{1}{2}, \frac{1}{2}\right]\right)$. Table 2 summarises the aliasing energy ratios up to level five for our sample filters. The ratio is significantly lower for all combined complex transforms shown in the lower part of the table; it is especially zero at level one. The Haar filter and its dual-tree version exhibit a high aliasing because it is badly localised and does not fulfil condition (7). On the other hand, again the filters $\mathrm{BW}_{m}$ and $\mathrm{BL}_{m}$ get of course less shift dependent as their order $m$ increases. We can conclude that all constructed filters with appropriate support property perform well so that we derived a general design method for shift invariant complex filters with perfect reconstruction. 


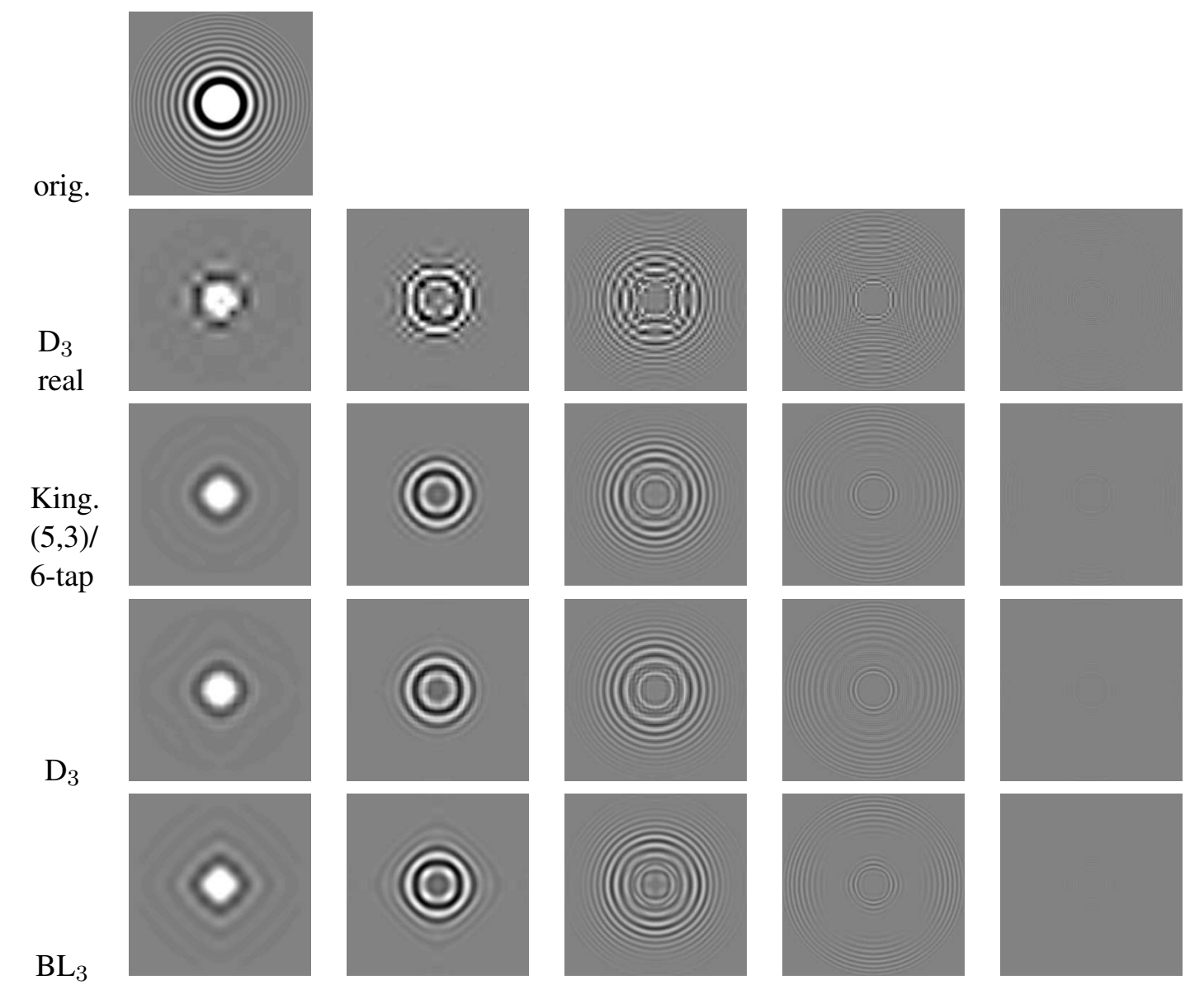

Figure 11: subband information of the real and complex transforms: contribution of low-pass and high-pass from level four to one

To illustrate the behaviour for the two-dimensional transform with respect to rotation invariance, we show the synthesis contributions of the different levels $S_{l}^{4}, S_{h}^{4}, \ldots, S_{h}^{1}$ for a rotationally symmetric image in Fig. 11. For each component, only the coefficients of the appropriate two resp. six directional subbands are passed to the respective inverse transform. As a result, the fully decimated real wavelet transform in the second row shows heavy blocky artifacts and aliasing. The complex transforms in the further rows look a lot better, especially for the same basis filter $\mathrm{D}_{3}$, but still the resulting reconstruction components are not rotationally invariant, see e.g. the high-pass at levels two and three. But according to [16], this may result from the two diagonal subbands having higher centre frequencies than the other four. The level four high-pass components in the second column rather show a diamond shape than a circle.

\section{Application to Signal Classification}

We intend to classify two different types of data, namely physiological heart patient data as in [26] and texture image rows as described in [19]. The problems 'heart5', 'heart6' and 'heart7' stand for the detection of ventricular tachycardia with real patient data for three different patients. Typical examples of curves from the two classes we want to distinguish are shown in Fig. 12. 


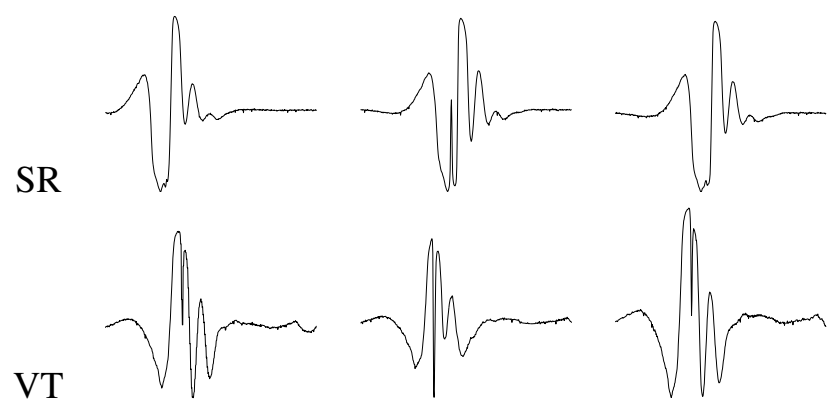

Figure 12: two-class problem (heart beats: sinus rhythm (SR) and ventricular tachycardia(VT))

class 1
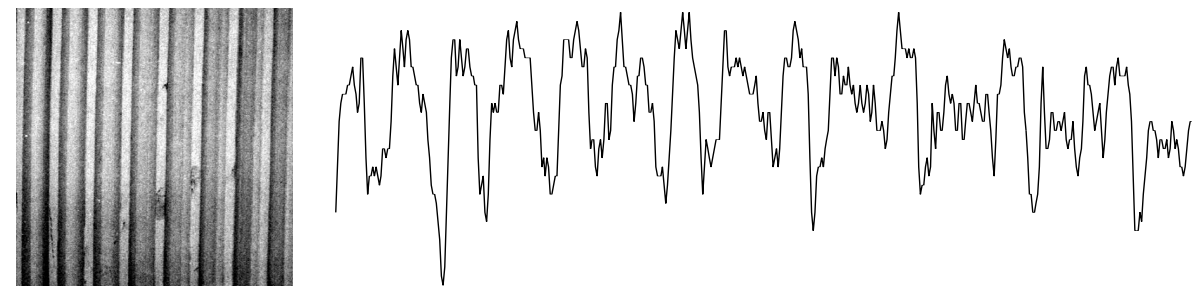

class -1
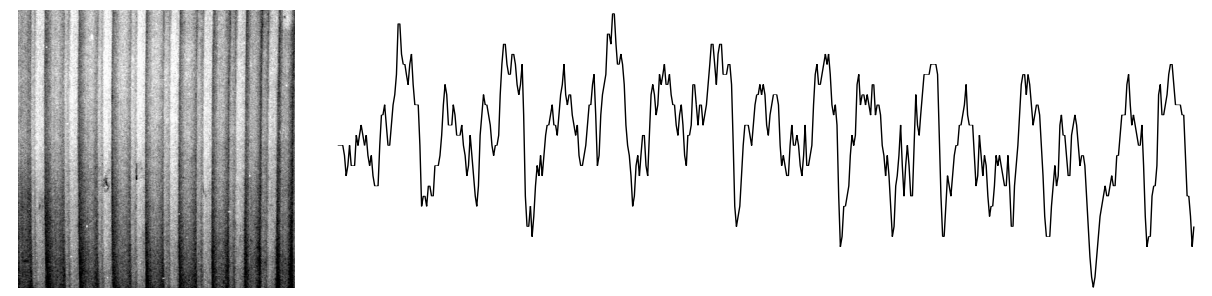

Figure 13: texture sample: linearly rescaled images and exemplary rows

Our problem 'misc $2-$ misc3' is a texture line classification problem. In contrast to the heart data, the typical curves depicted in Fig. 13 have a bad localisation in the time domain. As the previous signals they consist of 512 values.

The classification setup is illustrated in Fig. 14: As usual it includes a feature extraction step which reduces the number of features of each signal to prevent the curse of dimensionality and a subsequent classifier applied to the extracted features. For the feature extraction we use a wavelet transform (likewise critically sampled, non-subsampled or as dual-tree complex transform) in the filtering step and compute the 2-norm (energy) of the wavelet coefficients at each of the $d$ decomposition levels. These $d$ energy values represent our features. Note that the 2 -norm has, by the Parseval identity, the advantage that no back transform of the Fourier coefficients from the frequency to the time domain is necessary. Other norms may be used as well, but for our classification problem the influence of the norm was marginal. For the feature extraction, we also examine the ideal filter with support property supp $H_{0}\left(e^{2 \pi i \omega}\right)=\left[-\frac{1}{4}, \frac{1}{4}\right]$ which can be implemented by the Fourier transform. As our classifier serves a hard margin Support Vector Machine (SVM), see [28].

The classification results are given in Table 3. The data are separated into a training and a test set to evaluate the classification performance. In the 'trn shifted' problem versions, the test data instead consists of all distinct shifts of all training signals to evaluate shift invariance or all shifts of all test signals for 'tst shifted' to combine both issues. For 'trn shifted' and the transform without subsampling, no results are given because the feature vectors to classify are just the same as the training vectors. Consequently, the test error has to be zero. 


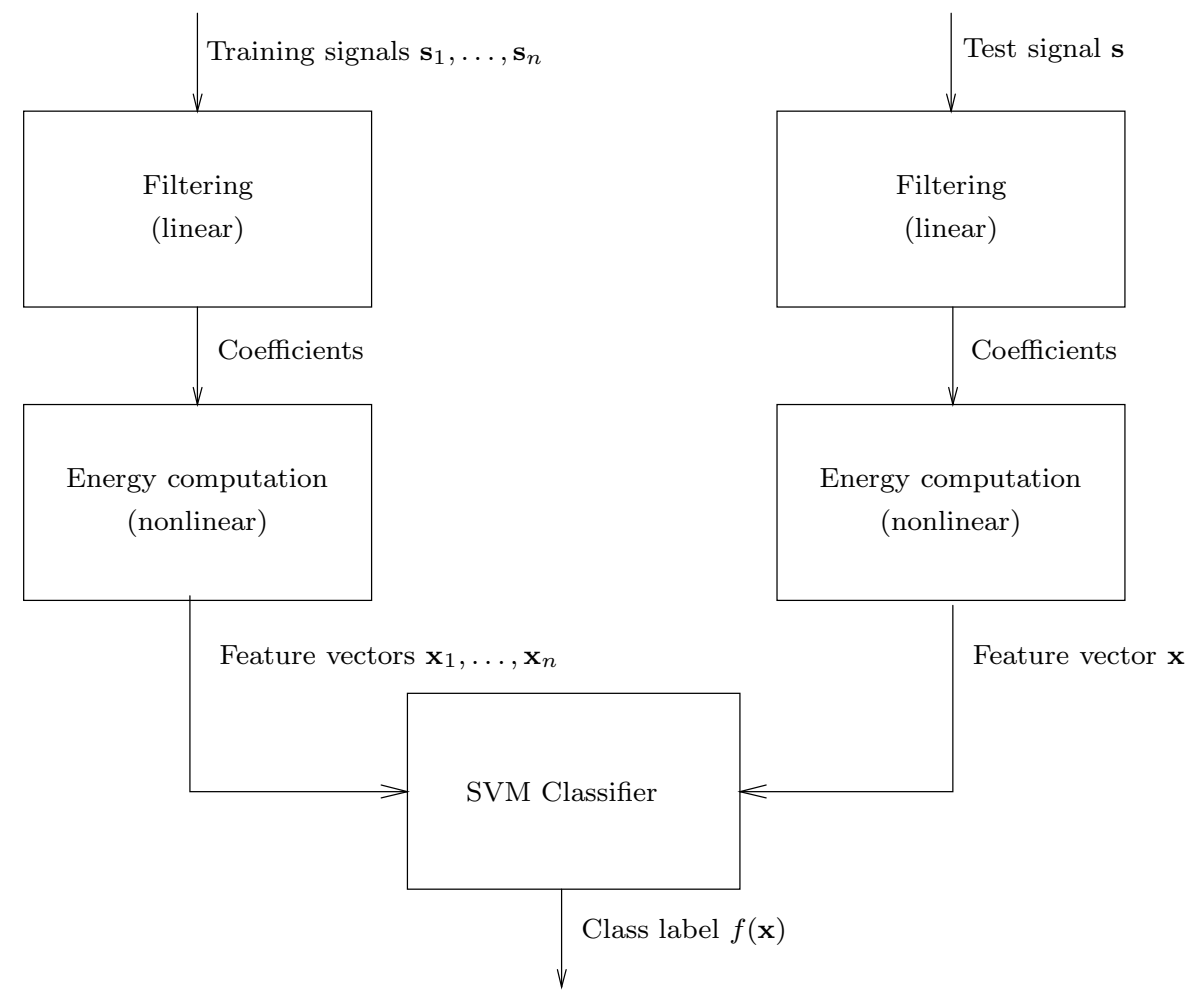

Figure 14: classification setup

\begin{tabular}{|c|c|c|c|c|c|c|c|c|c|c|c|c|c|}
\hline \multicolumn{2}{|r|}{ problem } & 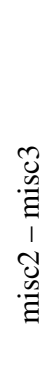 & 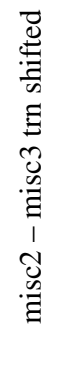 & 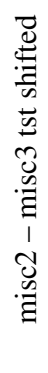 & 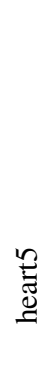 & 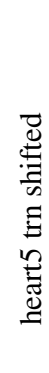 & 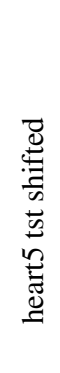 & 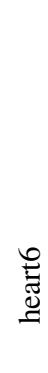 & 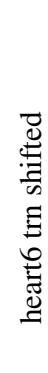 & 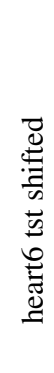 & 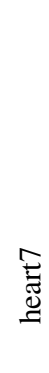 & 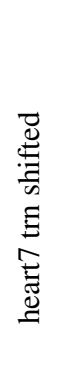 & 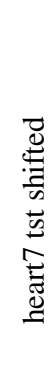 \\
\hline critically & $\mathrm{H}$ & 44 & 45 & 50 & 19 & 38 & 42 & 0 & 40 & 42 & 3 & 42 & 47 \\
\hline sampled & $\mathrm{D}_{3}$ & 49 & 34 & 36 & 9 & 41 & 36 & 6 & 55 & 54 & 13 & 23 & 30 \\
\hline no & $\mathrm{H}$ & 24 & - & 24 & 0 & - & 0 & 38 & - & 38 & 22 & - & 22 \\
\hline subsampling & $\mathrm{D}_{3}$ & 24 & - & 24 & 0 & - & 0 & 6 & - & 6 & 16 & - & 16 \\
\hline \multirow{2}{*}{ Kingsbury [16] } & $(5,3) / 6$-tap & 23 & 0 & 25 & 0 & 3 & 1 & 25 & 16 & 26 & 19 & 0 & 18 \\
\hline & $(9,7) / 14$-tap & 31 & 0 & 32 & 0 & 0 & 0 & 13 & 11 & 19 & 19 & 0 & 19 \\
\hline \multirow{6}{*}{$\begin{array}{c}\text { complex } \\
\text { (frequency domain) }\end{array}$} & $\mathrm{H}$ & 52 & 26 & 40 & 0 & 30 & 20 & 6 & 38 & 44 & 6 & 15 & 30 \\
\hline & $\mathrm{D}_{3}$ & 21 & 3 & 25 & 0 & 11 & 6 & 44 & 28 & 40 & 16 & 0 & 18 \\
\hline & $\mathrm{BW}_{3}$ & 25 & 0 & 26 & 0 & 0 & 0 & 19 & 15 & 21 & 19 & 0 & 22 \\
\hline & $\mathrm{BW}_{11}$ & 16 & 0 & 16 & 0 & 0 & 0 & 13 & 0 & 13 & 25 & 0 & 25 \\
\hline & $\mathrm{BL}_{1}$ & 24 & 0 & 26 & 0 & 2 & 1 & 34 & 26 & 30 & 16 & 0 & 17 \\
\hline & $\mathrm{BL}_{3}$ & 22 & 0 & 22 & 0 & 0 & 0 & 13 & 0 & 13 & 25 & 0 & 25 \\
\hline \multicolumn{2}{|c|}{ Fourier } & 0 & - & 0 & 0 & - & 0 & 13 & - & 13 & 50 & - & 50 \\
\hline
\end{tabular}

Table 3: classification error of a hybrid signal classifier for different filters in percent 
As a result, expectedly, the fully decimated wavelet transform is not able to well discriminate between the signal classes, particularly for the translated heart signals. But the most important observation is that the complex transform really succeeds in achieving approximate shift invariance. The classification error for the 'shifted' problems is definitely lower than that of the critically sampled real transform. Moreover, the results for the complex filters are comparable with the computationally expensive totally translation invariant transform without subsampling.

As the data for problem 'misc2 - misc3' show a highly periodic structure, the classification error decreases with the filter size and translation invariance is an important issue. Here the Fourier features perform best. For 'heart5', evidently, classification performance is highly dependent on the shift sensitivity so that all shift insensitive transforms perform well. The two further 'heart' problems are more complicated: Different filters, here, e.g. $\mathrm{H}$ and BL, are most successful whereas the Fourier features fail completely for 'heart7'.

\section{Conclusion}

We have worked out and applied Kingsbury's idea of dual-tree filter banks in the frequency domain where it can be based on standard wavelets. Concerning translation and rotation invariance these complex transforms behave much better than their critically sampled counterparts and show a performance at least as good as Kingsbury's specially designed filters. Of course our computation in the frequency domain involves (real) FFTs such that with respect to the arithmetic complexity it can only compete with real filter banks in the time domain involving filters of moderate length. We have applied the dual-tree filter banks in the feature extraction step for a classification problem. The feature extraction process and the subsequent classification benefit from an appropriate adaptation of the wavelet to the problem at hand. This votes for a whole library of dual-tree complex wavelet filter banks which is available based on the known wavelet filters if we work in the frequency domain.

In view of these properties, an extension to the classification application would be to adapt the complex filters to the classification problem, which means to the data and the classifier at hand. 


\section{References}

[1] C. P. Bernard. Discrete wavelet analysis for fast optic flow computation. Technical Report RI415, Centre de Mathématiques Appliquées, École Polytechnique, February 1999.

[2] G. Beylkin, R. Coifman, and V. Rokhlin. Fast wavelet transforms and numerical algorithms. Communications on Pure and Applied Mathematics, pages 141-183, 1991.

[3] C. Blatter. Wavelets - Eine Einführung. Vieweg, 2nd edition, 2003.

[4] R. R. Coifman and D. L. Donoho. Translation-invariant de-noising. In A. Antoniadis and G. Oppenheim, editors, Wavelets and Statistics, volume 103 of Lecture Notes in Statistics, pages 125-150. Springer, New York, 1995.

[5] R. R. Coifman and M. V. Wickerhauser. Entropy-based algorithms for best basis selection. IEEE Transactions on Information Theory, 32:712-718, March 1992.

[6] Z. Cvetković and M. Vetterli. Oversampled filter banks. IEEE Transactions on Signal Processing, 46(5):1245-1255, May 1998.

[7] I. Daubechies. Ten Lectures on Wavelets. Society for Industrial and Applied Mathematics, Philadelphia, PA, USA, 1992.

[8] P. F. C. de Rivaz and N. G. Kingsbury. Complex wavelet features for fast texture image retrieval. In Proc. IEEE Int. Conf. on Image Processing, 1999.

[9] D. L. Donoho. De-noising by soft-thresholding. IEEE Transactions on Information Theory, 41(3):613-627, 1995.

[10] F. C. A. Fernandes, I. W. Selesnick, R. L. C. van Spaendonck, and C. S. Burrus. Complex wavelet transforms with allpass filters. Signal Processing, 83(8):1689-1706, August 2003.

[11] B. Forster, T. Blu, and M. Unser. A new family of complex rotation-covariant multiresolution bases in 2d. In A. Aldroubi, A. F. Laine, and M. Unser, editors, Wavelet Applications in Signal and Image Processing X, Proceedings of the SPIE Conference on Mathematical Imaging, 2003.

[12] M. Frigo and S. G. Johnson. FFTW: An adaptive software architecture for the FFT. In Proc. 1998 IEEE Intl. Conf. Acoustics Speech and Signal Processing, volume 3, pages 1381-1384. IEEE, 1998. http://fftw.org.

[13] A. Gottscheber and G. Steidl. On a family of orthogonal wavelets on the quincunx grid. In W. Haußmann, K. Jetter, and M. Reimer, editors, Advances in Multivariate Approximation, pages 175-184. Wiley-VCH, Berlin, 1999.

[14] N. G. Kingsbury. The dual-tree complex wavelet transform: a new technique for shift invariance and directional filters. In Proc. of the IEEE Digital Signal Processing Workshop, August 1998.

[15] N. G. Kingsbury. Image processing with complex wavelets. Phil. Transactions Royal Society London A, 357:2543-2560, September 1999.

[16] N. G. Kingsbury. Complex wavelets for shift invariant analysis and filtering of signals. Journal of Applied and Computational Harmonic Analysis, 10(3):234-253, May 2001. 
[17] N. G. Kingsbury and J. F. A. Magarey. Wavelet transforms in image processing. In Proc. First European Conference on Signal Analysis and Prediction, pages 23-34. ICT Press, 1997.

[18] S. Mallat. A Wavelet Tour of Signal Processing. Academic Press, London, 1999.

[19] J. Neumann, C. Schnörr, and G. Steidl. Feasible adaptation criteria for hybrid wavelet large margin classifiers. Technical Report TR-02-015, Dept. of Mathematics and Computer Science, University of Mannheim, December 2002.

[20] A. V. Oppenheim and R. W. Schafer. Discrete-Time Signal Processing. Signal Processing Series. Prentice Hall, Englewood Cliffs, NJ, USA, 1989.

[21] J. Portilla and E. P. Simoncelli. A parametric texture model based on joint statistics of complex wavelet coefficients. International Journal of Computer Vision, 40(1):49-70, 2000 .

[22] N. Saito. Local Feature Extraction and Its Application Using a Library of Bases. PhD thesis, Department of Mathematics, Yale University, December 1994.

[23] I. W. Selesnick. Hilbert transform pairs of wavelet bases. IEEE Signal Processing Letters, 8(6):170-173, June 2001.

[24] E. P. Simoncelli, W. T. Freeman, E. H. Adelson, and D. J. Heeger. Shiftable multiscale transforms. IEEE Transactions on Information Theory, 38(2):587-607, March 1992. MIT Media Laboratory Vision and Modeling Technical Report No.161.

[25] G. Strang and T. Nguyen. Wavelets and Filter Banks. Wellesley-Cambridge Press, Wellesley, 1996.

[26] D. Strauß and G. Steidl. Hybrid wavelet-support vector classification of waveforms. Journal of Computational and Applied Mathematics, 148:375-400, 2002.

[27] M. Unser. Texture classification and segmentation using wavelet frames. IEEE Transactions on Image Processing, 4(11):1549-1560, 1995.

[28] V. Vapnik. The Nature of Statistical Learning Theory. Springer, New York, 1995.

[29] M. V. Wickerhauser. Adapted Wavelet Analysis from Theory to Software. A. K. Peters, Ltd., Wellesley, MA, 1994. 